\title{
Von performativen Äußerungen zum Performative Turn. Performativitätstheorien zwischen Sprach- und Medienparadigma
}

\author{
Lars Gertenbach
}

Online publiziert: 10. Dezember 2020

(C) Der/die Autor(en) 2020

Zusammenfassung Der Begriff der Performativität hat in den Sozial- und Kulturwissenschaften der letzten Jahre eine beachtliche Karriere aufzuweisen, bleibt aber inhaltlich oftmals unbestimmt. Gleiches gilt für den proklamierten Performative Turn. Ausgehend von aktuellen Debatten um das Performativitätskonzept, wie sie etwa im Anschluss an die Schriften von Karen Barad und Michel Callon geführt werden, sollen diese Diskussionen im Beitrag genauer sortiert und einer kritischen Reflexion unterzogen werden. Er beginnt zunächst mit einer Rekonstruktion der Sprechakttheorie von John Austin und der kulturwissenschaftlichen Aneignung des Begriffs der Performativität, bevor er sich anschließend eingehender der soziologischen Theorie zuwendet. Im Fokus stehen hierbei Pierre Bourdieu und Judith Butler, die als Gegenpositionen zu Barad und Callon begriffen werden können. Um die Differenz dieser beiden Varianten des Performative Turns genauer zu fassen, wird schließlich mithilfe neuerer Medientheorien zwischen zwei maßgeblichen Theoriemodellen unterschieden: einem sprachtheoretischen und einem medientheoretischen. Dadurch ist es möglich, Missverständnisse und Ungenauigkeiten in der bisherigen Auseinandersetzung aufzudecken und den Innovationsgehalt sowie die Schwachstellen der unterschiedlichen Positionen genauer abzuschätzen.

Schlüsselwörter Performativität · Performative Turn · Soziologische Theorie · Kulturtheorie · Michel Callon · Karen Barad · Pierre Bourdieu · Judith Butler • John Austin

\footnotetext{
L. Gertenbach $(\triangle)$

Fachbereich Gesellschaftswissenschaften, Fachgebiet Soziologische Theorie, Universität Kassel, Nora-Platiel-Str. 5, 34109 Kassel, Deutschland

E-Mail: lars.gertenbach@uni-kassel.de
} 


\title{
From performative utterances to the performative turn. Theories of performativity between the paradigm of language and media
}

\begin{abstract}
The concept of performativity has had a remarkable career in social and cultural theory in recent years, but its content often remains vague. The same holds true for the proclaimed performative turn. Starting from the current debates about the concept of performativity following, among others, the writings of Karen Barad and Michel Callon, the paper sets out to systematize and critically examine these discussions in greater detail. It begins with a reconstruction of John Austin's speech act theory and the adaptation of the concept of performativity by cultural theory, before turning to sociological approaches. The focus here lies on Pierre Bourdieu and Judith Butler, who can be understood as counter-positions to Barad and Callon. To grasp the difference between these two strands of the performative turn, the article draws on recent media theories to distinguish two theoretical paradigms: one rooted in language theory and one in media theory. This distinction makes it possible to reveal some misunderstandings and inaccuracies in the current debate and to assess the merit as well as the shortcomings of the respective positions more accurately.
\end{abstract}

Keywords Performativity · Performative turn - Social theory $\cdot$ Cultural theory · Michel Callon · Karen Barad · Pierre Bourdieu · Judith Butler · John Austin

\section{Des énoncés performatifs au tournant performatif. Les théories de la performativité entre paradigme du langage et paradigme des médias}

Résumé Ces dernières années, le concept de performativité a connu une carrière remarquable dans les sciences sociales et culturelles mais ce qu'il désigne demeure souvent vague. Il en va de même du « tournant performatif » qui est proclamé. Les discussions actuelles sur le concept de performativité, inspirées par exemple par les écrits de Karen Barad et Michel Callon, sont ici mises en ordre et soumises à une réflexion critique. Pour ce faire, l'article revient sur la théorie des actes de langage de John Austin et l'appropriation du concept de performativité par les sciences culturelles avant de se consacrer plus en détail à la théorie sociologique. L'accent est mis sur Pierre Bourdieu et Judith Butler qui peuvent être considérés comme des contradicteurs de Barad et Callon. Pour appréhender plus précisément la différence entre ces deux variantes du «tournant performatif », une distinction est établie à l'aide des nouvelles théories des médias entre deux principaux modèles théoriques : l'un basé sur la théorie du langage, l'autre sur la théorie des médias. Il est ainsi possible de déceler des malentendus et des imprécisions dans la discussion menée jusqu'à présent et d'évaluer plus précisément en quoi ces différentes positions innovent ainsi que leurs points faibles.

Mots-clés Performativité - Tournant performatif - Théorie sociologique · Théorie de la culture $\cdot$ Michel Callon · Karen Barad · Pierre Bourdieu · Judith Butler · John Austin 


\section{Einleitung}

Der Begriff der Performativität hat sich in den Sozial- und Kulturwissenschaften in den letzten Jahren zu einem äußerst populären Schlagwort entwickelt. Ursprünglich der linguistischen Sprechakttheorie entstammend, findet er sich mittlerweile in wirtschafts-, kultur-, organisations- und techniksoziologischen Arbeiten ebenso wie in Studien zu Geschlecht, Sport und Körperlichkeit oder in Forschungen zu Ritualen, zu Vergemeinschaftung und zu populistischer Politik. Weil er sich dabei für eine Vielzahl von soziologischen Theoriepositionen als anschlussfähig erwiesen hat, ist es fast folgerichtig zur Ausrufung eines Performative Turns gekommen (vgl. Gonquergood 1989; Martschukat und Patzold 2003; Medina 2010). Mit dieser Behauptung aber gehen zwei weitreichende Annahmen einher, die keineswegs unumstritten sind: erstens, die einer breitenwirksamen Aufnahme dieses Konzepts in die Forschungshaltung der entsprechenden Disziplinen; sowie, zweitens, die einer grundlegenden epistemischen Neuorientierung dieser Fächer. Auch die Metapher des Turns selbst unterstreicht diesen weitreichenden Anspruch. Denn sie setzt diese Forschungen in Tradition zu den wissenschaftsgeschichtlich wirkmächtigen Umbrüchen des Linguistic und des Cultural Turns.

$\mathrm{Ob}$ man diesen Annahmen zustimmen mag oder nicht, unstrittig ist in jedem Fall, dass der zunächst recht eng gefasste sprachwissenschaftliche Begriff der Performativität eine beachtliche Karriere in den Sozial- und Kulturwissenschaften hingelegt hat. Für seinen Aufstieg waren mindestens zwei günstige Rahmenbedingungen mitentscheidend: Zum einen spielt innerwissenschaftlich eine Rolle, dass die soziologische Forschung immer mehr von einem handlungs- oder kommunikationstheoretischen auf ein praxistheoretisches bzw. praxeologisches Vokabular umgestellt hat (vgl. Schäfer 2016). Das Konzept der Performativität konnte so zu einer Art Klammer um recht heterogene Theoriepositionen werden, sodass der Aufstieg jener Theoriepositionen zugleich zur Stärkung dieses Konzepts beigetragen hat. Obwohl der Performative Turn nicht mit der Etablierung praxistheoretischer Positionen in eins fällt, bewirken letztlich beide, dass Fragen des Vollzugs, der Inszenierung und der Wirkmächtigkeit - und das heißt eben der Performanz - von sozialen Praktiken stärker in den Fokus rücken. ${ }^{1}$ Diese Orientierung am Vollzug mag zwar auf den ersten Blick marginal erscheinen. Mit ihr ist aber, wie Sybille Krämer in ihrer Auseinandersetzung mit dem Performativitätskonzept betont hat, ,eine Akzentverschiebung verbunden, die nicht nur für die Sprachanalyse, sondern für den gesamten Bereich kulturwissenschaftlichen Forschens anregend und impulsgebend werden kann.“ (Krämer 2002, S. 344). ${ }^{2}$

Zum anderen spielt für die Anschlussfähigkeit und Plausibilität des Konzepts eine Rolle, dass die Orientierung an Performanz(en) zu einem wesentlichen Merkmal

\footnotetext{
${ }^{1}$ Zur inhaltlichen Nähe von Praxistheorie und Performativitätskonzept vgl. Hempfer und Volbers (2011) sowie Kertscher und Mersch (2003).

2 Diese Priorisierung des Vollzugs ist die Grundlage des von Krämer als „Performanz-Modell“ bezeichneten Zugangs zur sprachtheoretisch zentralen Frage nach dem Verhältnis von Sprache und Sprechen. Ihm gegenüber stehen die als „Zwei-Welten-Modell“ bezeichneten Positionen von Saussure, Searle, Chomsky und Habermas, die für eine Priorisierung der Form bzw. des Systems oder Regelwerks eintreten (Krämer 2001).
} 
zeitgenössischer Kultur geworden ist (vgl. Fischer-Lichte 1998). Ersichtlich ist dies bereits daran, dass Metaphern aus dem Bereich des Theaters (wieder) vermehrt in der Soziologie und in populären Zeitdiagnosen Einzug erhalten haben. Sie werden herangezogen, um den Aufstieg einer „Ökonomie der Aufmerksamkeit“ (Franck 1998) zu beschreiben, auf die gestiegene Bedeutung von (Selbst-)Inszenierungen hinzuweisen oder eine Kultur des Gespielten und Oberflächlichen zu bemängeln - sei es in Bezug auf politische Kommunikation, neue und alte Medien, ritualhafte Vergemeinschaftung oder die Selbstdarstellung auf Online-Plattformen. ${ }^{3}$ Durch ihre Nähe zu solchen Theatermetaphern erweisen sich die Begriffe der Performanz und der Performativität als höchst anschlussfähig für die Beschreibung unterschiedlicher Tendenzen zeitgenössischer Vergesellschaftung - etwa wenn Arbeit zum Projekt, Kultur zum Event oder Handlungen zur Performance umgedeutet werden.

Eines der ersten Werke, das eine Logik der Performanz in unterschiedlichen gesellschaftlichen Sphären im Aufstieg begriffen sieht und das den Begriff zugleich zur Leitfigur einer Zeitdiagnose macht, ist Jean-Francois Lyotards La condition postmoderne aus dem Jahr 1979 (Lyotard 1986). Lyotard beschreibt dort, wie die Ordnungen und die Logik des Wissens in postmodernen Gesellschaften durch die zunehmende Ausbreitung des Performativitätskriteriums transformiert werden. ${ }^{4}$ Das an Input/ Output-Relationen ausgerichtete, auf Leistung zielende Modell der Performanz führt, so Lyotard, dazu, dass die Steigerung von Effizienz zum allgemeinen Legitimationskriterium unterschiedlichster gesellschaftlicher Sphären und Institutionen wird, an dem sich Wissenschaft und Erziehung ebenso messen lassen müssen wie Wirtschaft und Technik. Eine etwas anders gelagerte, aber ebenso prominente Stellung nimmt die These der Performanzkultur auch in aktuelleren Arbeiten ein. So heißt es etwa in einem Schlüsselabschnitt von Andreas Reckwitz' Zeitdiagnose der Gesellschaft der Singularitäten: „Die singularistische soziale Praxis nimmt grundsätzlich die Struktur einer Aufführung an, so dass Performativität ihr zentrales Charakteristikum ist. [...] Singuläre Entitäten werden nicht primär instrumentell ge- und vernutzt (zweckrationales Handeln) oder normativ behandelt (normatives Handeln), sondern bieten sich im Modus der Performativität dar" (Reckwitz 2017, S. 72; Hervorh. i. Orig.).

Vor diesem Hintergrund ist es nicht verwunderlich, dass dem Begriff der Performativität eine zentrale Rolle in der gegenwärtigen soziologischen Forschung zukommt. Und auch die Ausrufung eines Performative Turns ist angesichts die-

\footnotetext{
3 Vgl. exemplarisch Bauman (2009, S. 73 ff.) für die Dominanz des Ästhetischen und Inszenierten insbesondere in Bezug auf Individualität und (neue) Vergemeinschaftung, für einen allgemeinen Überblick Volbers (2014) sowie zur Theatermetaphorik Pefanis (2017).

${ }^{4}$ Ich beziehe mich in dieser Formulierung auf das französische Original, weil die Metapher der Ausbreitung treffender die in dieser Zeitdiagnose enthaltene Transformationsdynamik charakterisiert als die englische und deutsche Übersetzung. Denn Lyotards im Text mehrfach wiederkehrende Formulierung ,la prévalence du critère de performativité" (Lyotard 1979, S. 78) erhält in diesen Übersetzungen einen anderen Klang, weil „prévalence“ als „predominance“ oder als „Vorrang“ (Lyotard 1986, S. 140) übersetzt wird. Für die allgemeine Verbindung von Postmoderne und Performativität s. Lyotard (ebd., S. 122); für eine genauere Aufschlüsselung der Konsequenzen dieser Umstellung auf Performativität ebd., S. $179 \mathrm{ff}$.
} 
ser Konvergenz von Theorieentwicklung und Zeitdiagnostik durchaus naheliegend. ${ }^{5}$ Was auf den ersten Blick als Stärke erscheinen mag, kann bei genauerem Hinsehen aber zugleich als problematisch begriffen werden. Denn gerade die hohe Anschlussfähigkeit und die oftmals lose und assoziative Begriffsverwendung lassen die Befürchtung entstehen, dass die konzeptionelle Schärfe des Begriffs im Moment seines Erfolges verloren zu gehen droht - so spricht Klaus Hempfer von der ,fundamental ,rhizomatische[n] Struktur“ des Konzepts“, in deren Folge es zu „kontradiktorischen Bestimmungen“ komme (2011, S. 38). Am prominentesten findet sich diese Kritik in jenen Teilbereichen der Soziologie, die sehr intensiv an diesen Begriff angeschlossen haben. Das beste Beispiel hierfür ist die neuere Wirtschaftssoziologie. Nach einer Phase der Proliferation von performativitätstheoretischen Studien ist es dort zu einer gewissen Relativierung des Konzepts gekommen. Fabian Muniesa bemängelt etwa: ,the notion of performativity (or the idea of the performative) has been used in a variety of ways which are often unrelated and perhaps even contradictory, in reference to speech, theatre, efficacy, and so forth. There is, as far as I can say, no integrative, consensual, coherent view on this and we are therefore in the somewhat uncomfortable, but quite fertile ground of ambiguity." (Muniesa 2014, S. 7; analog auch: Santos und Rodrigues 2009) Ganz ähnlich monieren auch Ivan Boldyrev und Ekaterina Svetlova in einem Überblickswerk zur wirtschaftssoziologischen Performativitätsforschung: ,the proliferation of empirical studies diluted the term ,performativity " often reducing it just to a ubiquitous catch-all concept." (Boldyrev und Svetlova 2016, S. 2) Derlei Kritik an der zwar produktiven, aber eben dennoch diffusen Verwendung des Performativitätsbegriffs ist trotz einer gewissen Pauschalisierung instruktiv, weil sie auf grundbegriffliche Unklarheiten und sozialtheoretische Leerstellen dieser Forschung hinweist. ${ }^{6}$ Insbesondere Boldyrev und Svetlova legen dabei nahe, dass an die Stelle theoretischer Überlegungen eine idiosynkratische Theoriesprache ohne eingehendere theoretische Reflexion getreten ist (ebd.).

Eine Forderung, die oftmals auf solche oder ähnliche Kritikpunkte folgt, lautet, die Überdehnung des Begriffs durch Engführungen oder Präzisierungen zu

\footnotetext{
5 Damit soll nicht behauptet werden, dass die Begriffe Performanz und Performativität deckungsgleich sind. Üblicherweise besitzt Performanz einen stärkeren Bezug zur Theatermetapher und bezieht sich auf einen allgemeinen Handlungsmodus der Aufführung, der Inszenierung und der Darstellung (vgl. exempl. Carlson 2004 als Beispiel einer nur auf den Begriff der ,performance“ und nicht auf den der Performativität setzenden Publikation). Performativität hingegen ist enger mit dem sprachtheoretischen Modell der Hervorbringung verbunden und bezieht sich auf den wirklichkeitserzeugenden Effekt sprachlicher Benennung oder auf performative Äußerungen im engeren Sinne. Obwohl diese Bedeutungsdimensionen viele Diskussionen und Verwendungsweisen in den Debatten um Performativität prägen, sind sie gerade in sozial- und kulturwissenschaftlichen Positionen umstritten (vgl. Bal 2001) - insbesondere dann, wenn sie eng mit dem Performative Turn verknüpft sind, wie etwa bei Butler oder Callon. Dementsprechend wird diese Diskussion im Folgenden zumindest implizit mitverhandelt. Im Sinne einer klareren Begrifflichkeit verwende ich fortan aber ausschließlich den Begriff der Performativität und spreche von dem Performative Turn in Bezug auf die sozial- und kulturtheoretische Wende der Performativitätsforschung.

6 Zum Überblick über die Kritik an der Performativitätsdebatte in der neueren Wirtschaftssoziologie vgl. Sparsam (2018) und Vosselman (2014).
} 
vermeiden. ${ }^{7}$ Weil eine Eingrenzung des Konzepts aber Gefahr läuft, die breite Anschlussfähigkeit des Begriffs zu verlieren und so die wesentlichen Errungenschaften des Performative Turns preiszugeben, soll eine solche Strategie im Folgenden ausdrücklich nicht verfolgt werden. Stattdessen soll die Unklarheit des Begriffs als Chance begriffen werden, um noch einmal auf die Grundannahmen dieses Konzepts zu reflektieren und auch solche Positionen in die Diskussion mit einzubeziehen, die im Streben nach Eingrenzung oftmals allzu schnell marginalisiert werden. Erst dann kann nämlich gezeigt werden, dass der Debatte - in der neueren Wirtschaftssoziologie ebenso wie in der allgemeinen Soziologie - ein zentrales Missverständnis zugrunde liegt, das dazu führt, dass einige Ansätze inhaltlich verkannt und damit in ihrer möglichen Bedeutung für diese Debatte unterschätzt werden. Weil sich aber gerade diese Positionen zur Korrektur der Probleme dieses Forschungsansatzes eignen, ist es notwendig, noch einmal genauer auf die Entwicklung dieser Debatte zu schauen.

Das wesentliche Ziel der folgenden Ausführungen besteht demnach in einer kritischen Reflexion der Performativitätsforschung und des Performative Turns. Im Fokus stehen dabei aktuelle Debatten um die Grenzen und die Erweiterung des Performativitätskonzepts, wie sie vor allem entlang der Schriften von Karen Barad und Michel Callon geführt werden. Um deren Beitrag für eine Weiterentwicklung dieser Debatte einschätzen zu können, ist es nötig, noch einmal den Ausgangspunkt des Konzepts sowie dessen kulturwissenschaftliche Aneignung in den Blick zu nehmen. Ich widme mich folglich zuerst der Sprechakttheorie von John L. Austin und dessen Unterscheidung zwischen konstativen und performativen Sprechakten (Abschnitt 2). Dieser Einstiegspunkt wird auch deshalb gewählt, weil die Auseinandersetzung um den Performativitätsbegriff auf verschiedenen Lesarten von Austin beruht, mit denen unterschiedliche Forschungszugänge verbunden sind. Auf dieser Basis kann schließlich die jüngere Debatte um den Performative Turn in den Blick genommen werden (Abschnitt 3). Die Aufnahme des Performativitätsbegriffs in den Sozial- und Kulturwissenschaften erzeugt eine gewisse Spannung zu Austin und den sprachwissenschaftlichen Weiterentwicklungen der Sprechakttheorie. Sie geht nicht nur mit einer abweichenden Lesart Austins einher, sondern führt gerade in der Soziologie zu ganz unterschiedlichen Theorieanschlüssen. Dies zeigt sich beispielhaft an Pierre Bourdieu und Judith Butler, die hier als zwei Varianten einer Soziologie der Performativität vorgestellt werden (Abschnitt 4). Der Fokus meiner Darstellung liegt dabei weiterhin auf dem jeweiligen Anschluss und der Kritik an Austin, weil sich hieraus ein wichtiger Kontrast gegenüber einer anderen Perspektive auf Performativität ergibt, die in der jüngeren Debatte entweder noch nicht hinreichend berücksichtigt oder nur mit erheblichen Missverständnissen rezipiert wurde. Als prototypische Positionen können hier Karen Barad und Michel Callon gelten, deren Annahmen zunächst aus ihrer Kritik an Butler und Bourdieu rekonstruiert werden, weil dadurch die Differenz zu den skizzierten sprachwissenschaftlichen und soziologischen Debatten deutlicher zutage tritt (Abschnitt 5). Dabei zeigt sich, dass die Diskussion

\footnotetext{
7 Stellvertretend für die Wirtschaftssoziologie steht hier Donald MacKenzies Versuch der Differenzierung verschiedener Intensitätsgrade von Performativität (MacKenzie 2006). Prototypisch für die allgemeine Performativitätsdebatte ist Hempfer (2011).
} 
von Barad und Callon nicht nur eine kritische Auseinandersetzung mit den theoretischen Prämissen des Performative Turns erlaubt. Sie eröffnet auch einen Weg, den Performativitätsbegriff anders als in den dominanten Theoriepositionen zu fassen. Um diese Differenz zwischen zwei mitunter konträren Varianten des Performative Turns genauer benennen zu können, unterscheide ich unter Rückgriff auf neuere Medientheorien abschließend zwischen zwei paradigmatischen Zugängen zum Konzept der Performativität: einem sprachtheoretischen und einem medientheoretischen (Abschnitt 6). Diese Differenz soll dabei behilflich sein, die Missverständnisse in der bisherigen Rezeption dieser Positionen aufzudecken und zugleich den Innovationsgehalt und die Herausforderungen genauer abschätzen zu können, die von ihnen ausgehen. Auf dieser Grundlage wird es schließlich möglich sein, die Performativitätsdebatte mit anderen zeitgenössischen Theorieentwicklungen und Debatten der Sozial- und Kulturwissenschaften in Verbindung zu setzen und dementsprechend in ihrer Bedeutung genauer einzuordnen.

\section{John Austin und die kollabierende Unterscheidung von performativen und konstativen Äußerungen}

Im Unterschied $\mathrm{zu}$ vielen anderen Konzepten verfügt der Performativitätsbegriff über einen klar benennbaren Ursprung, der in den daran anschließenden Debatten auch als steter Referenzpunkt wachgehalten wird. Den Initialpunkt dieser Debatte bildet John Austins 1955 im Rahmen der William James Lectures in Harvard gehaltenen Vorlesungen How to do things with words (Austin 1962; dt.: 2002). ${ }^{8}$ Einen zentralen Stellenwert erhalten diese Vorlesungen bereits durch ihre grundlegende Kritik an der klassischen Philosophie der Sprache. Zum einen setzt Austin gegen die primäre Beschäftigung mit der Logik und Struktur der Sprache auf eine Analyse der Sprachhandlung, d.h. der konkreten Sprechakte. Und zum anderen weist er die Annahme zurück, ,,von Interesse [für die Philosophie der Sprache; L. G.] seien ausschließlich Äußerungen, die Tatsachen wiedergeben oder Situationen wahr oder falsch beschreiben“ (Austin 1986, S. 305). Ihren Rang als Gründungstext der Performativitätsforschung erhalten diese Vorlesungen letztlich daraus, dass Austin in der Analyse dieser Sprechakte den Begriff der performativen Äußerung einführt. Dieser soll eine besondere Klasse von Äußerungen bezeichnen, die, so Austin, sowohl von der Sprachtheorie als auch der Philosophie weitgehend ignoriert oder vorschnell als unwesentlich oder uninteressant abgestempelt worden seien (ebd.).

Kennzeichnend für performative Äußerungen ist vor allem, dass sie nicht in das klassische Modell von Aussagen passen, weil sie keine Feststellungen oder Behauptungen sind, die als wahr oder falsch begriffen werden können. „An der Oberfläche haben sie das Aussehen - oder jedenfalls die grammatische Politur - von ,Aussagen “;

\footnotetext{
8 Dies gilt vor allem wirkungsgeschichtlich für die Debatte um den Performativitätsbegriff. In Austins Werk finden sich bereits einige den William James Lectures vorausgehende Überlegungen. Sie betreffen zum einen Vorlesungen in Oxford, die er von 1952 bis 1954 unter dem Titel Words and deeds gehalten hat, und zum anderen den 1946 veröffentlichten Aufsatz Other minds, der inhaltlich bereits die Performativitätsdebatte vorbereitet und den Begriff, wenn auch eher lose, verwendet - etwa in der Diskussion der sprachlichen Struktur von Versprechungen (Austin 1946, S. 186f.).
} 
nichtsdestoweniger zeigt genaueres Hinsehen, daß sie keine Äußerungen darstellen, die ,wahr" oder ,falsch“ sein könnten." (Austin 2002, S. 35) Was sie vielmehr auszeichnet, ist, dass sie durch die Äußerung hindurch etwas vollziehen, was mehr ist als nur eine sprachliche Bezeichnung. Ihr zentraler Wert liegt nicht in der Referenz, sondern im hervorbringenden Vollzug: ,sie beschreiben keine Tatsachen, sondern sie schaffen soziale Tatsachen“ (Wirth 2009, S. 11). Um diese Differenz genauer zu benennen, unterscheidet Austin zwischen performativen und konstativen Äußerungen. Während Letztere Aussagen über etwas sind und damit etwas konstatieren, das zumindest prinzipiell auf seinen Wahrheitsgehalt hin überprüft werden kann, läuft diese Zuschreibung für die nun gesonderte Klasse der performativen Äußerungen ins Leere. „Die konstatierende Äußerung, unter dem bei Philosophen so beliebten Namen der Aussage, hat die Eigenschaft, wahr oder falsch zu sein. Demgegenüber kann die performative Äußerung niemals eins von beiden sein, sie hat vielmehr eine eigene Funktion: sie wird zum Vollzug einer Handlung gebraucht. Eine solche Äußerung tun, ist die Handlung vollziehen, eine Handlung, die man vielleicht kaum, zumindest nicht mit gleicher Präzision, auf andere Weise vollziehen könnte.“ (Austin 1968, S. 140; Hervorh. im Orig.) Austins Beispiele der Schiffstaufe, der Trauung oder der Wette machen deutlich, dass sich hier das Geäußerte (die Aussage) nicht von der Äußerung (dem Akt der Aussage) trennen lässt: ,Jeder würde sagen, daß ich mit diesen Äußerungen etwas Bestimmtes tue (natürlich nur unter passenden Umständen); dabei ist klar, daß ich mit ihnen nicht beschreibe, was ich tue, oder feststelle, daß ich es tue; den Satz äußern heißt: es tun.“ (Austin 2002, S. 29) Performative Äußerungen haben damit kein äußerliches Verhältnis zur Wirklichkeit, sondern sie vollziehen die Wirklichkeit, die sie beschreiben, selbst mit und bringen sie so erst hervor. Worum es Austin also geht, ist, dass diese Aussagen durch die Äußerung selbst etwas tun; etwas, das - unter passenden Umständen glücken kann oder eben nicht (Austin 1986, S. 309 ff.). Kennzeichnend für performative Äußerungen ist somit, dass an die Stelle der Unterscheidung von wahr und falsch die Frage nach dem Gelingen oder Misslingen der Äußerung tritt. Weil diese aber von den genauen Umständen und Kontextbedingungen des Sprechens abhängt, wird eine rein an ihrem sprachlichen Gehalt orientierte, sprachimmanente Prüfung von Äußerungen problematisch.

Schon diese wenigen Bemerkungen lassen erahnen, welche Sprengkraft diese Idee für das Verständnis von Sprache haben kann - selbst auf dieser noch sehr basalen Ebene der Identifikation einer gesonderten Klasse von Äußerungen. Weil performative Akte nicht im klassischen Sinne wahr oder falsch sein können, hat dies Folgen für Korrespondenzmodelle von Sprache, deren Geltung relativiert oder sogar generell infrage gestellt wird. Stimmt man dem zu, dann ist Sprache weder das, was klassische Denotations- und Repräsentationstheorien hierunter verstehen, noch ist sie bloßes Instrument und Medium für Mitteilungen über die Welt (die richtig sein können oder nicht). Vielmehr kann das Sprechen selbst unter bestimmten Umständen eine in die Welt eingreifende Handlung sein - und zwar im bzw. durch den Akt des Sprechens selbst. „Wenn wir performative Äußerungen tun, dann kann man ganz vernünftiger Weise sagen, daß wir ,Handlungen vollziehen“ [...].“ (Austin 2002, S. 42f.) Diese Verquickung von Sprechen und Handeln unterläuft bzw. sprengt das klassische Sprachmodell insofern, als sie das Handlungsmoment von Sprache 
anders akzentuiert und erweitert: „Wir handeln also nicht nur, dadurch dass wir sprechen, sondern wir handeln auch, indem wir sprechen." (Krämer 2003, S. 19; Hervorh. i. Orig.)

Ohne Zweifel ergibt sich an dieser Stelle bereits eine Vielzahl von Anschlussmöglichkeiten für die Kultur- und Sozialwissenschaften. Zum Verständnis der Eigenheiten des Performative Turns ist allerdings wesentlich, dass Austin in seinen Vorlesungen hierbei nicht stehen bleibt, sondern letztlich einen anderen Weg einschlägt. Entscheidend ist, dass er zunächst versucht, die Differenz von performativen und konstativen Sprechakten weiter zu entfalten. Dabei soll der aus der Beschäftigung mit dem Performativen gewonnene Handlungsaspekt als wesentliches Unterscheidungskriterium dienen. Austin startet also damit, die Differenz von performativen und konstativen Äußerungen herzuleiten als ,Unterscheidung zwischen Tun und Sagen“ (Austin 2002, S. 65). Obwohl sich dies zunächst als geeignete und auch begrifflich hinreichend klare Differenz zu erweisen scheint, entwickelt er jedoch allmählich Zweifel, die von einer Vorlesung zur nächsten schließlich immer größer werden. Angedeutet werden sie noch in der gleichen Vorlesungssitzung, die mit dem Satz endet: „Vielleicht ist der Unterschied zwischen beiden gar nicht so groß.“ (ebd., S. 73) Erkennbar werden sie jedoch vor allem ab der darauffolgenden fünften Vorlesung, in der Austin die Befürchtung formuliert, ,daß unsere ursprüngliche, versuchsweise Unterscheidung zwischen konstativen und performativen Äußerungen auf die eine oder andere Weise zusammenbrechen könnte" (ebd., S. 75). In den weiteren Sitzungen schwindet die Hoffnung in die eindeutige Abgrenzbarkeit von konstativen und performativen Äußerungen schließlich immer weiter, weil die eigentlich klar zugeordneten Eigenschaften beider Äußerungstypen immer mehr ineinander verschwimmen. So heißt es:

Unser Ausgangspunkt war, daß wir einen Gegensatz zwischen performativen und konstativen Äußerungen vermutet haben. Wir haben aber hinreichende Anzeichen dafür gefunden, daß Äußerungen beider Typen, nicht nur die performativen, verunglücken können; weiter, daß auch die performativen Äußerungen nicht nur glücken müssen, sondern der Forderung unterliegen, den Tatsachen zu entsprechen oder doch in einer je nach Fall verschiedenen Beziehung zu den Tatsachen zu stehen, ganz wie das für allem Anschein nach konstative Äußerungen typisch ist. (ebd., S. 109)

Weil sich die Zweifel an der Tragfähigkeit der Leitunterscheidung immer mehr häufen, legt Austin sie schließlich beiseite und beginnt, sie durch die Unterscheidung von lokutionären, illokutionären und perlokutionären Bestandteilen des Sprechakts zu ersetzen - bis er sie in der letzten, zwölften Vorlesung vollends aufgibt (ebd., S. 168). Die nun dreiteilige Unterscheidung verzichtet auf eine Klassifizierung unterschiedlicher Sprechakte. Sie führt stattdessen die Idee einer graduellen Differenz ein, in der mal dieser, mal jener Aspekt im Zentrum steht, ohne dass die anderen dabei verschwinden würden. Für Austin ergibt sich daraus der Vorteil einer differenzierteren und weniger rigiden Terminologie, in der schließlich nicht länger die eine gegen die andere Aussagenklasse ausgespielt werden muss.

Diese drastische Zurücknahme des kurz zuvor eingeführten Konzepts ist eigentümlich und in der Forschung auch bereits ausgiebig diskutiert worden (vgl. Krämer 
2003; Loxley 2007; Searle 1989). Besonders bemerkenswert ist dabei, dass sich daraus trotz allem eine höchst produktive Forschungsrichtung entwickelt hat. Denn die Geschichte des Performativitätsbegriffs hätte mit diesen Vorlesungen genauso gut beginnen wie an ihr Ende kommen können. Weil dies im Werk von Austin und in der weiteren Sprechakttheorie etwa bei John Searle auch tatsächlich der Fall ist, stellt sich die Frage, warum ausgerechnet die Kultur- und Sozialwissenschaften diesen Begriff aufgegriffen haben.

\section{Von performativen Äußerungen zum Performative Turn}

Obwohl Austins Vorlesungen den Begriff der Performativität überhaupt erst prominent machen, lassen sie dessen Einführung im Rückblick eher als eine Art Verlegenheitslösung erscheinen. Die in den Vorlesungen schließlich vollzogene Hinwendung zur Trias von lokutionären, illokutionären und perlokutionären Bestandteilen von Sprechakten bestärkt diesen Eindruck noch und legt nahe, dass sich all diese Überlegungen im Vorfeld einer angestrebten begrifflichen Präzisierung bewegen. Genau diese Lesart wird schließlich in der weiteren Entwicklung der Sprechakttheorie bei Austin und vor allem bei Searle vertreten. Der Begriff der Performativität erscheint hier zusammen mit der Idee der Sonderstellung performativer Äußerungen als überwundener Zwischenschritt, der in der neuen Nomenklatur der Sprechakttheorie gewissermaßen aufgehoben ist. So greift Searle nicht mehr direkt auf die Unterscheidung zwischen dem Konstativen und dem Performativen zurück. Im Fokus stehen stattdessen spezifischere Diskussionen zur Sonderstellung performativer Verben (Searle 1989) und allgemeine Überlegungen zur ,,performance of an illocutionary act" (Searle 1969, S. 29). ${ }^{9}$

Dass der Begriff in den Sozial- und Kulturwissenschaften trotzdem attraktiv und anschlussfähig erscheint, hat mehrere Gründe, die sich hier bereits stichpunktartig benennen lassen. Erstens bestärkt er die in den Sozial- und Kulturwissenschaften seit den 1960er-Jahren immer prominentere Zurückweisung von Repräsentations- und Abbildungsmodellen von Sprache und Wissen (vgl. Pickering 1995, S. 182; Krämer 2011, S. 181). Er bietet sich als veritables Gegenmodell an, um die Vorstellung einer strikten Trennung von Sache und Aussage, Welt und Wort oder Natur und Kultur infrage zu stellen. Zweitens erlaubt die Verquickung von Sprache und Kontext eine Herauslösung der Sprachanalyse aus dem alleinigen Zuständigkeitsbereich der Sprachwissenschaften. Damit verbunden bietet der Begriff drittens ein gewichtiges Argument, um die Vorherrschaft der formalen Logik in der Analyse von Wissen und Sprache zu problematisieren und - analog etwa zur Metaphorologie - den produktiven Charakter des Rhetorischen, also etwa von Sprachbildern und Metaphern, zu betonen. Weil der Begriff zudem erlaubt, Sprache stärker als Tätigkeit zu begreifen, ergeben sich viertens zahlreiche Anschlussmöglichkeiten an praxistheoretisch argumentierende Kommunikations- und Interaktionstheorien. Freilich kann all dies auch ohne den Begriff der Performativität erforscht und diskutiert werden. Weil das

\footnotetext{
9 Einen Überblick über zentrale Positionen der philosophischen bzw. sprachtheoretischen Debatte bieten Loxley (2007) und Wirth (2009).
} 
Konzept über die Sprachtheorie hinaus aber zum Inbegriff eines auf Vollzug gerichteten praxistheoretischen Denkens geworden ist, konnte es sich zum ,umbrella term" für zahlreiche Forschungen entwickeln - selbst dort, wo es nicht unmittelbar um Sprache geht.

Diese verschiedenen Aspekte liefern bereits stichhaltige Gründe für den Erfolg des Konzepts. Für die Verschiebung des Performativitätsbegriffs von der Analyse sprachlicher Äußerungen zum Grundbegriff von Sozialität schlechthin spielt aber noch ein weiterer Faktor eine wichtige Rolle. Die sozial- und kulturwissenschaftliche Forschung beginnt nämlich allmählich, eine andere Lesart der Vorlesungen von Austin ins Spiel zu bringen. Entscheidend ist dabei die Frage, ob die Weiterentwicklung hin zur Begriffstrias lokutionär, illokutionär und perlokutionär als Fort- oder Rückschritt begriffen werden muss (vgl. Müller 2015, S. 69). Aus Sicht zahlreicher kultur- und sozialwissenschaftlicher Ansätze ist die Fortschrittslesart deshalb problematisch, weil mit der Abkehr vom Performativitätsbegriff zugleich die Kritik am klassischen Sprachmodell verloren geht. Denn Austins Hinweise auf die Mängel des bestehenden Sprachmodells lassen sich zum Ausgangspunkt einer sozial- und kulturwissenschaftlichen Erweiterung der Sprachtheorie machen. Aus der Kritik an der fehlenden begrifflichen Trennschärfe wird so ein Lob auf das Subversions- und Irritationspotential des Begriffs - das ja bei Austin selbst bereits angedeutet ist, wenn er etwa über den philosophischen Aussagebegriff schreibt: „Was wir im Hinblick auf Feststellungen oder Aussagen und daher auch mit Bezug auf das Beschreiben und Berichten tun müssen, ist, sie von ihrem hohen Sockel herunterzuholen und zu erkennen, daß sie ebensosehr Sprechakte sind wie jene anderen, die wir erwähnt und unter der Bezeichnung ,performative " besprochen haben." (Austin 1986, S. 324) Im Unterschied zur sprachwissenschaftlichen Weiterführung schließt die sozial- und kulturwissenschaftliche Aneignung von Austin stärker an derartige Aussagen an und versucht, die philosophischen und sozialtheoretischen Konsequenzen der Reformulierung der Sprachtheorie herauszuarbeiten.

Aus Sicht der Kulturwissenschaften hat sich das Konzept der Performativität somit auch deshalb als tragfähig erwiesen, weil es die Unterscheidung, auf deren Basis es eingeführt wurde, zunichtemacht. Austins Scheitern, die Differenz zwischen konstativen und performativen Äußerungen aufrechtzuerhalten, wird so zu seinem eigentlichen Verdienst, weil gerade die Entgrenzung des Konzepts der Performativität zu seiner weiteren Verbreitung beiträgt. Die Unmöglichkeit der kategorialen Trennung der performativen von anderen Äußerungen wird als Anstoß genommen, jede geschlossene Sprachtheorie zurückzuweisen. Und weil Austin daran zweifelt, dass sich überhaupt noch eine klar abgrenzbare Klasse konstativer Äußerungen bestimmen lässt, wird betont, dass selbst die vermeintlich konstative Frage der Adäquanz und Richtigkeit einer Aussage stets von Aufführungs- und Gelingensbedingungen durchkreuzt wird. Diese grundlegende Kritik an dem Topos des Konstativen motiviert schließlich zu einer gänzlich anderen Lesart von Austin. Sie hat ihren Ausgangspunkt in Shoshana Felmans Studie Le scandale du corps parlant aus dem Jahr 1980 (Felman 2003), in der sie vorschlägt, die Vorlesungen selbst als Inszenierung, d.h. als Performance zu lesen - und nicht wie üblich allein als konstatives Unterfangen begrifflicher Präzisierung: „Although Austin was constantly asking what we $d o$ with (or through) what we say, the history of ideas has never inquired into 
what Austin was doing with what he was saying." (ebd., S. 49; Hervorh. i. Orig.) Dabei zeigt sich, dass Austin die Unhaltbarkeit der Unterscheidung zwischen konstativen und performativen Äußerungen nicht nur feststellt, d.h. konstatiert, sondern ihr Scheitern inszeniert, d.h. ,performed“ (ebd., S. 48 f., 80 ff.). Unter diesen Vorzeichen steht die Begriffstrias lokutionär, illokutionär und perlokutionär für einen Rückfall der Sprechakttheorie in den Modus des Konstativen.

Die sozial- und kulturwissenschaftliche Aneignung des Performativitätsbegriffs erfolgt - wie mit Bezug auf Felman vor allem Krämer (2001, S. 150f.) und Wirth (2009) betonen - also durchaus im Bewusstsein, an eine wesentliche, wenn auch vielleicht etwas verborgene Dimension des Programms von Austin anzuschließen. Inhaltlich maßgeblich ist dabei das Argument, dass die Bedingungen des Gelingens von sprachlichen Handlungen nicht (allein) in der Sprache selbst liegen. Damit aber läuft der Performative Turn im Kern auf eine Soziologie des Performativen hinaus. Deren genaue Ausgestaltung lässt sich insbesondere im Rekurs auf zwei hierfür paradigmatische Positionen erschließen: der Pierre Bourdieus und Judith Butlers. Obwohl beide jeweils andere Schlussfolgerungen aus der Beschäftigung mit der Sprechakttheorie ziehen und auch unterschiedliche Akzentuierungen vornehmen, bilden sie gerade in dieser Komplementarität den soziologischen Diskurs um Performativität ab.

\section{Soziologien des Performativen - Pierre Bourdieu und Judith Butler}

Durch die praxistheoretische Grundhaltung Bourdieus nehmen Fragen des Vollzugs und der kulturellen Inszenierung sozialer Ordnung eine zentrale Rolle in seinem Werk ein. Gleichwohl gehört der Begriff der Performativität nicht zu den offensichtlichen Leitbegriffen seiner Sozialtheorie. Eine Auseinandersetzung mit Austins Vorlesungen sowie der Sprechakttheorie als solcher findet sich dennoch in verschiedenen Schriften, allen voran in Was heißt sprechen? Zur Ökonomie des sprachlichen Tausches (Bourdieu 2015). Für Bourdieu ist Austins Ansatz hochgradig anschlussfähig an die Soziologie, weil dort in der Analyse von Sprechakten ausdrücklich auf die Kontextbedingungen des Sprechens hingewiesen wird. Problematisch erscheint ihm jedoch, dass es bei Austin meist bei einem beiläufigen Hinweis auf die Umstände des Sprechens bleibt. Zwar finden sich immer wieder Verweise darauf, dass das Gelingen performativer Aussagen von ,,speziellen“, „,bestimmten“ oder ,,passenden Umständen“ abhängt (Austin 2002, S. 29, 36f., 101), eine genauere Analyse bleibt im Rahmen der Sprechakttheorie jedoch aus. Für Bourdieu übergeht Austin damit die Einsicht, dass ,die performative Aussage als Akt der Setzung sozio-logisch nicht unabhängig von der Institution bestehen kann, der sie ihre Daseinsberechtigung verdankt“ (Bourdieu 2015, S. 81). So kann für ihn Austins Analyse ,innerhalb der Grenzen der Sprachwissenschaft zu keinem Schluss kommen. Die magische Wirkung dieser Setzungsakte ist nicht von der Existenz einer Institution zu trennen, die die Bedingungen (für Akteure, Orte, Zeitpunkte usw.) bestimmt, die erfüllt sein müssen, damit die Magie der Worte wirken kann“ (ebd., S. 80). Für Bourdieu ist damit klar, dass die Theorie des Performativen überhaupt nur im Rekurs auf die Soziologie ihr eigentliches Versprechen einlösen kann. Er kritisiert demzufolge vor allem den 
Versuch, die performative Kraft der Sprache in der Sprache selbst zu verankern, d.h. eine genuin sprachliche Substanz (performative Verben, spezifische grammatische Formen etc.) hierfür verantwortlich zu machen (Bourdieu und Wacquant 1996, S. 183). Dies erscheint ihm als „Ursprung jenes am deutlichsten bei Austin (oder später bei Habermas) zu beobachtenden Irrtums, der im Diskurs selber, das heißt in der eigentlichen sprachlichen Substanz [...] des Wortes den Ursprung seiner Wirkung zu entdecken meint. Der Versuch, die Macht sprachlicher Äußerungen sprachlich zu begreifen, die Suche nach der Ursache der Logik und der Wirkung der Sprache der Setzung in der Sprache selber, übersieht, dass die Sprache ihre Autorität von außen bekommt [...].“ (Bourdieu 2015, S. 101; Hervorh. i. Orig.)

Mit diesem Hinweis auf die außersprachlich fundierte Autorität der Sprache deutet sich bereits ein zentraler Aspekt in Bourdieus soziologischer Wendung der Theorie des Performativen an. Weil das Gelingen performativen Sprechens von Kontexten, d.h. von institutionellen Bedingungen abhängig ist, ergibt sich für ihn eine zwingende Verknüpfung mit der Frage nach Autorität und symbolischer Herrschaft, also eine Verbindung von Sprach- und Machtanalyse. Und weil die performative Aussage „einen ausdrücklichen Machtanspruch“ (ebd., S. 81f.) in sich trägt, ist genau diese Analyse Aufgabe der Soziologie des Performativen: „Damit macht die Suche nach dem sprachlichen Ursprung jener ,außersprachlichen Macht' des Diskurses der eigentlichen soziologischen Suche nach den Bedingungen Platz, die einem einzelnen Akteur, und mit ihm seinem Wort, eine derartige Macht verschaffen können." (ebd., S. 82)

Anders als etwa Habermas setzt Bourdieu in seiner Kritik der Sprechakttheorie somit von vornherein am Begriff der Performativität an. Sie läuft dabei auf eine Soziologie des Performativen hinaus, die auch als Social Turn der Sprachtheorie begriffen werden kann. Denn sie zielt weniger auf eine soziologische Ergänzung der Sprachtheorie als dass sie Austins Überlegungen zum Anlass nimmt, Sprachanalyse über soziologische Kategorien neu zu fundieren. Der Rückgriff auf Kategorien wie Macht und Autorität sowie die wiederkehrende Rückbindung der Sprachtheorie an die Frage der Institution sorgen dafür, dass das Soziale zur Erklärungsinstanz des Sprachlichen wird (ebd., S. 105). Im Werk Bourdieus selber hat dies schließlich einen durchaus paradoxen Effekt. Denn weil die Theorie des Performativen in eine allgemeinere Theorie symbolischer Herrschaft eingelassen ist, kommt der Begriff der Performativität selber in seinem Werk nur selten vor: „Die Frage der performativen Aussagen wird klarer, wenn man sie als einen Sonderfall der Wirkungen symbolischer Herrschaft begreift, die in jedem sprachlichen Tausch eine Rolle spielt." (ebd., S. 79) Bourdieu steht damit paradigmatisch für eine Soziologisierung des Performativen. Bezeichnend ist dabei auch, dass er in seiner Kritik an der Sprechakttheorie immer wieder die vermeintliche „Magie“ und das „Mysterium des Performativen“ (Bourdieu 1991, S. 106, 122) angreift. Denn seine eigenen Ausführungen zielen auf eine Entmystifizierung dieser besonderen Magie des Sprachlichen: „Tatsächlich üben Worte eine typisch magische Macht aus: sie machen sehen, sie machen glauben, sie machen handeln. Aber wie im Fall der Magie muß man sich fragen, worin das Prinzip dieses Vorgangs besteht; oder genauer welche die sozialen Bedingungen sind, die die magische Wirksamkeit der Worte möglich machen." (Bourdieu 1992, 
S. 83) Genau in diesem Punkt besteht eine zentrale Differenz zur zweiten hier als paradigmatisch verhandelten Soziologie des Performativen.

Im Unterschied zu Bourdieu nimmt der Begriff der Performativität bei Judith Butler auch im Gesamtwerk eine tragende Rolle ein. Ihre zentralen Argumente lassen sich gerade über die Kritik an Bourdieu gut erschließen, insbesondere an dessen Entmystifizierung und Soziologisierung der vermeintlichen Magie der Sprache. Bereits der Titel ihres Aufsatzes zu Bourdieu - „Performativity's social magic“ (Butler 1999) - zeigt dabei die Richtung an. ${ }^{10}$ Obwohl auch in Butlers Verständnis von Performativität Fragen der Macht und der Autorisierung von zentraler Bedeutung sind, distanziert sie sich von Bourdieu aufgrund dessen einseitiger Hinwendung zu den Kontextbedingungen des Sprechens. Für Butler geht damit nämlich der eigentliche Gewinn des Performativitätsbegriffs verloren: die Betonung der generativen Kraft des Sprachlichen. Bourdieus Rückbindung des Performativen an eine bereits bestehende soziale Autorität operiere letztlich mit einem zu einfachen Widerspiegelungsmechanismus: „he construes a mimetic relation between the linguistic and the social, rehabilitating the base/superstructure model whereby the linguistic becomes epiphenomenal“ (ebd., S. 122). Das Soziale wird damit in Bourdieus Performativitätskonzept nicht nur zum Grund performativen Sprechens, es scheint auch dem Sprechen ebenso wie dem Subjekt immer schon vorauszugehen. „,[A] speaker who declares a war or performs a wedding ceremony, and pronounces into being that which he declares to be true, will be able to animate the social magic' of the performative to the extent that that subject is already authorized or, in Bourdieu's terms, delegated to perform such binding speech acts“ (ebd., S. 122; Hervorh. i. Orig.).

Was genau ist hieran für Butler aber nun problematisch? Ihre Kritik richtet sich vor allem darauf, dass Bourdieus Programm der Entautonomisierung des Sprachlichen die Eigenlogik und Wirkmächtigkeit sprachlicher Benennungen ignoriert. Bedacht auf die Autonomie des Sozialen, begeht er damit in gewisser Weise den spiegelbildlichen Fehler wie die auf die Autonomie des Sprachlichen bedachten Sprechakttheorien: ,he fails to take account of the way in which social positions are themselves constructed through a more tacit operation of performativity." (ebd., S. 122) Dadurch wird Bourdieus Aneignung der Sprechakttheorie für Butler in zweierlei Hinsicht fragwürdig. Erstens baut sie auf einer zu statischen Vorstellung des Sozialen auf. Mit Bourdieus Versuch, das Gelingen der performativen Äußerungen als genuin sozio-logisches Problem umzudeuten, droht aus ihrer Sicht das im Begriff der Performativität zentrale Moment der Hervorbringung und damit auch des Neuen und Ereignishaften wieder verlorenzugehen. Und zweitens tendiert er dazu, den Mechanismus der Performativität auf die bloße Reproduktion präexistenter sozialer Strukturen, Positionen und Autoritäten zu reduzieren. Ihr eigener Ansatz versucht genau diesen Determinismus aufzubrechen: „If a performative brings about what it names, does it do this by itself, or does it proceed through a kind of citation or appropriation of ,authority " that effectively produces the effect of authority at deauthorized sites on the social map?" (ebd., S. 124; vgl. auch Eickelmann 2017, S. $53 \mathrm{ff}$.) Diese im Grunde rhetorische (und, zugegeben, recht umständlich formu-

${ }_{10}$ Diese Überlegungen finden sich auch im Schlusskapitel von Haß spricht. Zur Politik des Performativen (Butler 2006, S. 221-255). Vgl. zur Diskussion von Bourdieu und Butler auch Lovell (2000). 
lierte) Frage ist deshalb instruktiv, weil hierin zwei zum Verständnis von Butlers Position wesentliche Momente enthalten sind: der Aspekt der Zitation sowie der Hinweis, dass Autorität auch als Effekt oder Produkt performativen Sprechens begriffen werden kann. Beide Aspekte stehen sinnbildlich für die poststrukturalistische Wendung des Performativitätsbegriffs, die ihren Ausgangspunkt bereits in Derridas Auseinandersetzung mit Austin und der Sprechakttheorie hat (Derrida 1999, 2002).

Performative Äußerungen als Zitationen zu begreifen (Butler 1995, S. 124), impliziert für Butler (und Derrida), das Verhältnis von Kontext und Autorisierung anders zu denken und den Vorgang der Autorisierung als komplexeren und gebrocheneren Prozess zu begreifen. Der sprachlichen Logik der Zitation kommt dabei die Eigenschaft zu, einen Kontext gleichermaßen heranzuziehen und mit ihm zu brechen (Butler 2006, S. 231; Derrida 1999, S. 335, 339). Diese Simultaneität von Differenz und Wiederholung, für die der Begriff der „Iterabilität" ${ }^{\text {"11 }}$ einstehen soll, eröffnet für Butler eine Möglichkeit, der sprachlichen Eigenlogik des Performativen gerecht zu werden, ohne deren Angewiesenheit auf Kontexte gänzlich negieren zu müssen. Genau dies begründet ihre Differenz zu Bourdieu: „Seine konservative Erklärung des Sprechakts geht davon aus, daß die Konventionen, die die performative Äußerung autorisieren, bereits bestehen, und deswegen kann er den Derridaschen ,Bruch “ mit dem Kontext, den Äußerungen performativ herbeiführen, nicht erklären.“ (Butler 2006, S. 222f.)

In einer ähnlichen Weise vollzieht auch der zweite angesprochene Aspekt eine Abkehr von Bourdieu, die dennoch nicht zu einer Autonomisierung der Sprache im Sinne der Sprechakttheorie zurückführt. Auch hier kritisiert Butler im Rückgriff auf Derrida die Statik von Bourdieus Soziologie des Sozialen, wenn sie betont, dass performatives Sprechen als Autorisierung begriffen werden muss. Ein zentrales Argument betrifft dabei die spezifische Zeitlichkeit, in die das performative Sprechen eingelassen ist (Butler 2002, S. 117). ${ }^{12}$ Schon Derrida hatte am Beispiel der amerikanischen Unabhängigkeitserklärung gezeigt, dass dieser ,deklaratorische Akt“ (Derrida 2002, S. 124) eine Art retrospektive Vorgängigkeit erzeugt. Denn das spezifisch Performative dieser Unterzeichnung besteht darin, dass es das Volk als die unterzeichnende Entität eben nicht vertritt, sondern überhaupt erst hervorbringt:

[...] dieses Volk existiert nicht, nicht vor dieser Erklärung, nicht als solches.

Durch jene Unterzeichnung bringt es sich als freies und unabhängiges Subjekt, als möglicher Unterzeichner zur Welt. Die Unterschrift erfindet den Unterzeichner. Dieser kann sich erst dann zur Unterzeichnung ermächtigen, wenn er [...] mit seiner Unterzeichnung mittels einer wundersamen Rückkopplung ans Ende gekommen ist. (ebd., S. 124)

\footnotetext{
11 Vgl. Derrida (1999, S. 340f.) sowie Butlers Kommentar zum darin enthaltenen Konzept der Wiederholung (1997, S. 337, Anm. 9).

12 Butler kritisiert dementsprechend an Bourdieu auch genau diese Vernachlässigung des Zeitlichen in seiner Konzeption des Sozialen - zugunsten einer allzu statischen räumlichen Konzeption: „The domain of the social cannot be reduced to a spatialized context, in which' a temporalized habitus in general or the linguistic habitus in particular effects its rituals. For the question of how social positions are produced and reproduced will raise the question of the ,temporality " of positions themselves." (Butler 1999, S. 124).
} 
Der hierin aufgeworfene Aspekt der Zeitlichkeit findet sich bei Butler in der Betonung von Iterabilität und performativer Autorisierung wieder. Zusammen mit Derrida steht sie dabei für eine Theorielinie, die auf die generative Wirksamkeit von Sprache und Diskursen abstellt. Dabei begreift sie Performativität als ,eine spezifische Modalität der Macht als Diskurs“ (Butler 1997, S. 259). Die Berücksichtigung der Eigenlogik des Sprachlichen führt dazu, dass Performativität vor allem als spezifische Benennungsmacht verstanden wird, wodurch sich der Blick auf die materialisierenden Wirkungen von Diskursen richtet (ebd., S. 259). Aus diesem Grund erweist sich der Performativitätsbegriff bei Butler schließlich als Schlüsselkonzept ihres Werkes, der nicht nur sprach- und körpertheoretische Fragen verbindet, sondern diese auch mit ihrer Subjektivierungstheorie der Anrufung (Butler 2002) und einer politischen Theorie der Versammlung (Butler 2015) verknüpft. Im Vergleich zu Bourdieu verschiebt dies letztlich auch den Problemzusammenhang des Performativitätskonzepts. Er verläuft nun im Spannungsfeld von Diskurs und Materialität bzw. des Symbolischen und des Realen. ${ }^{13}$

Trotz aller Differenzen zwischen Bourdieu und Butler wird damit erkennbar, warum gerade diese beiden als paradigmatische Positionen einer Soziologie des Performativen begriffen werden können. Erstens sind in soziologischen Performativitätstheorien genau jene zwei Aspekte zentral, die bei Bourdieu und Butler am deutlichsten zum Vorschein kommen: der soziale Kontext sprachlicher Äußerungen sowie die Macht und Autorität des Symbolischen. Zweitens stehen sie sinnbildlich für die Spannweite der soziologischen Auseinandersetzung mit der Frage, wie das Verhältnis zwischen dem Sozialen und dem Sprachlichen gedacht werden kann. Und dank der praxistheoretischen Einbettung ihrer Positionen wird schließlich drittens bei beiden deutlich, dass Sprache in einer Soziologie des Performativen nicht (mehr) als geschlossenes System, sondern als komplexes Vollzugsmedium gedacht werden muss - auch wenn freilich strittig bleibt, ob Sprache dabei als Vollzugsmedium des Sozialen begriffen werden sollte.

\section{Posthumanistische Performativität - Karen Barad und Michel Callon}

Die als Soziologien des Performativen rekonstruierten Positionen lassen sich nun als Kontrastfolie für die Diskussion einer anderen Theorielinie nutzen. Diese findet ihre prominenteste Ausprägung in den Arbeiten von Karen Barad und Michel Callon. Obwohl beide in ihren Schriften ausdrücklich für ein neues Verständnis von Performativität eintreten, sind ihre Beiträge in der aktuellen Debatte bis jetzt nicht angemessen berücksichtigt worden. Übersehen wurde dadurch, dass sowohl von Barad als auch von Callon wichtige Impulse für eine Weiterentwicklung des Performativitätskonzepts ausgehen. Um diese aufzuzeigen, gilt es, die Differenzen zu den bisher rekonstruierten sprachtheoretischen und soziologischen Performativitätstheorien genauer zu eruieren. Dass Barad und Callon dabei bislang noch nicht

13 Letzteres gilt vor allem für die Lacan-Diskussion im siebten Kapitel von Körper von Gewicht (Butler 1997, S. 257 ff.). 
zusammen diskutiert wurden, ${ }^{14}$ kann durchaus überraschen, weil beide für eine ähnliche Position eintreten. So kritisieren beide die (gerade auch soziologische) Performativitätsforschung für ihren Reduktionismus, ihren Anthropozentrismus und ihre Diskurs- bzw. Sprachzentriertheit. Hinzu kommt, dass sie sich dazu eignen, einen idealtypischen Gegenentwurf zu den Positionen von Bourdieu und Butler zu skizzieren. Dieser Kontrast, so die Hoffnung, kann sichtbar machen, worin der Beitrag dieser Positionen besteht und warum er bislang häufig übersehen wurde.

Bei Barad steht vor allem Butler im Zentrum ihrer Diskussion des Performativitätskonzepts. In der Verknüpfung von Wissenschaftsforschung, feministischer Theorie und Neuem Materialismus tritt sie für eine Neubestimmung des Verhältnisses von Diskursivem und Materiellem unter dem Titel eines ,,agentiellen Realismus“ ein (Barad 2012a). Besondere Aufmerksamkeit hat dabei ihre Kritik an der Vorherrschaft des Sprachlichen und Kulturellen erfahren - ein Argument, das sich gegen die Dominanz des Linguistic und des Cultural Turns sowohl in der Wissenschaftsforschung wie auch in den Kulturwissenschaften und im Feminismus richtet: „Language has been granted too much power. [...] it seems that [...] lately every ,thing “ - even materiality - is turned into a matter of language or some other form of cultural representation.“ (Barad 2007, S. 132) Barads Schriften zielen auf die Etablierung eines ,posthumanistischen“ Verständnisses von Performativität (Barad 2003), dessen Kern die Erweiterung des Konzepts um die Dimension des Materiellen ist. An Butler kritisiert sie dementsprechend, dass diese trotz der praxistheoretischen Ausrichtung und aller Diskussion um Materialität(en) einem sprachtheoretischen und letztlich auch anthropozentrischen Ansatz verhaftet bleibt:

Leider setzt Butlers Theorie die Materie letztlich wieder als passives Produkt von Diskurspraktiken fest statt als einen aktiven Akteur, der am eigentlichen Prozess der Materialisierung teilhat. [...] Außerdem ist Butlers Theorie der Materialität auf eine Theorie der Materialisierung menschlicher Körper begrenzt, oder genauer auf die Konstruktion der Konturen des menschlichen Körpers. Wie ihre Lektüre der Materialität im Sinne von Foucaultschen regulativen Praktiken deutlich macht, sind darüber hinaus die Prozesse, die ihr wichtig sind, nur menschliche gesellschaftliche Praktiken (wodurch genau die Dichotomie zwischen Natur und Kultur wieder eingesetzt wird, die sie bestreiten will). (Barad 2012a, S. 39)

Diese Kritik zeigt, dass Performativität bei Barad weniger als Inbegriff sprachlicher Wirkmächtigkeit, sondern als grundlegendes Gegenmodell zum klassischen Repräsentations- und Abbildungsdenken gilt (Barad 2007, S. 28). Ihr Einsatzpunkt

\footnotetext{
14 Eine gewisse Ausnahme bildet ein Aufsatz zur Organisations- und Managementforschung (Gond et al. 2016), der fünf fundierende „Bausteine“ der Performativitätsforschung (,Foundational perspectives: the building blocks of performativity studies“) unterscheidet: (1) ,performativity as doing things with words“ (Austin); (2) „performativity as efficiency“ (Lyotard); (3) ,performativity as actors constituting the self“ (Derrida, Butler); (4) „,performativity as bringing theory into being“ (Callon, Latour, MacKenzie); (5) ,performativity as socio-materiality mattering“ (Barad). Diese Sortierung in dem unübersichtlichen Feld der Performativitätsforschung ist zweifellos instruktiv. Auch hier wird aber Callon (sowie Latour) nur sehr einseitig rezipiert, wodurch sein eigentlicher Beitrag in dieser Debatte und seine Ähnlichkeiten zu Barad übersehen werden.
} 
besteht dementsprechend zunächst in der Entwicklung eines anderen, aktiveren Verständnisses von Materialität bzw. eines anderen Modells des Prozesses der Materialisierung (Barad 2012a, S. 39) - ganz ähnlich wie Donna Haraway, an die sie sich hier anlehnt (Barad 2007, S. 41; Eickelmann 2017, S. 32 ff.).

Darüber hinaus kritisiert sie an Butler deren Verständnis von Iterabilität. Für Barad drückt sich darin eine Priorisierung der Sprache aus, weil so einzig sprachlich und anthropozentrisch konzipierte Akte als ereignishaft begriffen und mit Veränderungspotenzial ausgestattet werden. An die Stelle des Konzepts der iterativen Zitation setzt sie das der ,,iterativen Intraaktivität“ (Barad 2012b, S. 49, Anm. 24), das nicht die Wirkmächtigkeit der Sprache, sondern die gegenseitige Verschränkung unterschiedlicher Entitäten zum Ausdruck bringen soll. ${ }^{15}$ Vor allem soll es dabei behilflich sein, Wandel nicht auf sprachliche Resignifizierungsprozesse zu verkürzen (Hoppe und Lemke 2015, S. 264). So bleibt das klassische Modell der Performativität für Barad unidirektional, weil der Wirkungszusammenhang zwischen Sprache und Materialität nur eine Richtung zu kennen scheint. ${ }^{16}$ Das verhindere aber die Entwicklung eines angemessenen Verständnisses von Materialität, in dem diese mehr ist als „entweder etwas Gegebenes oder als bloße Wirkung menschlichen Tätigseins“ (Barad 2012a, S. 97). Demgegenüber tritt Barad für einen Performativitätsbegriff ein, in dem auch die Wirkungsmacht des Materiellen inbegriffen ist: „Eine agentiellrealistische Ausarbeitung von Performativität räumt der Materie auf entscheidende Weise ihren Anteil als aktiver Teilhaber am Werden der Welt, an ihrer fortlaufenden Intraaktivität ein.“ (ebd., S. 13)

Obwohl dieser Argumentation gelegentlich eine etwas zu einfache Lesart des Poststrukturalismus zugrunde liegt, ${ }^{17}$ trifft sie doch einen wichtigen Punkt. Denn gerade poststrukturalistisch inspirierte Forschungen münden häufig in eine kulturalistische Überhöhung des Sprachlichen und Diskursiven. Und weil auch bei Butler trotz aller Orientierung an Körperlichkeit und Materialität die Benennungs- und Klassifikationsmacht der Sprache im Zentrum steht, hat Barads Kritik durchaus ihre Berechtigung. Doch obwohl sie wichtige Hinweise auf die Probleme diskurstheoretischer Performativitätskonzepte gibt, bleibt ihre Neubestimmung des Performativitätsbegriffs insgesamt recht abstrakt. Aus diesem Grund ist es hilfreich, ihre Überlegungen mit denen von Callon zu verbinden.

Ebenso wie Barad geht es Callon um eine Kritik an einem einseitig sprachtheoretischen und kulturalistischen Verständnis von Performativität. Obwohl er hierbei auf eine Argumentation zurückgreift, die bereits in den zum Teil mit Bruno Latour verfassten Gründungstexten der Akteur-Netzwerk-Theorie anzutreffen ist (Callon

\footnotetext{
15 ,The neologism, intra-action“ signifies the mutual constitution of entangled agencies. That is, in contrast to the usual ,interaction, ' which assumes that there are separate individual agencies that precede their interaction, the notion of intra-action recognizes that distinct agencies do not precede, but rather emerge through, their intra-action." (Barad 2007, S. 33).

16 Derart begreift Butler Materialität etwa als ,unkenntlich gewordene Wirkung der Macht“ und schreibt mit Bezug auf das Zitationskonzept: „In diesem Sinne kann Materialisierung als die sedimentierende Wirkung einer regulierten Wiederholbarkeit beschrieben werden." (Butler 1997, S. 345 f., Anm. 33).

17 Barad selbst legt an anderer Stelle (2007, S. 192) eine differenziertere Lesart von Butler vor. Eine deutliche Kritik an der einseitigen Butler-Lektüre und der damit verbundenen „Neugründungsgeste“ von Barad findet sich bei Sara Ahmed (2008); für eine ausführlichere Diskussion vgl. Eickelmann (2017).
} 
und Latour 2006; Gertenbach und Laux 2018), nimmt der Performativitätsbegriff erst in seinen wirtschafts- und techniksoziologischen Studien eine zentrale Rolle ein (Callon 1998, 2004, 2007). Sein eigener Beitrag zur Performativitätsdebatte erschließt sich sowohl aus seinen Fallstudien zu Finanzmärkten wie auch aus einigen theoriegeschichtlichen Kommentaren. Zunächst erscheint er dabei als typischer Vertreter des Performative Turns, denn die Attraktivität des Konzepts ergibt sich auch für ihn aus der Kritik am Repräsentationsdenken und aus der Zurückweisung eines am Modell des Konstativen ausgerichteten Sprachverständnisses. Wie viele andere zieht er hieraus die Konsequenz, dass Aussage und Kontext miteinander verbunden sind und Sprache als eine Form der Handlung begriffen werden muss: ,there is no language; there are only acts of language“ (Callon 2007, S. 318). Auch seine Kritik an Austin scheint sich zunächst in eine ähnliche Richtung zu bewegen wie die von Bourdieu. So schreibt er gegen die sprachliche Autonomiethese: ,The obvious limit of Austin's work is that his analysis does not depart from discourse per se, as his work on the different categories of verbs and performativities shows. Consequently, he can explain neither the force of statements nor their meaning." (ebd., S. 352, Anm. 6) Warum er dennoch eine andere Position als Bourdieu einnimmt, wird im weiteren Fortgang dieser Formulierung sichtbar, wenn er sich sowohl von subjekttheoretischen als auch von klassisch sozialtheoretischen Erweiterungen von Austin distanziert:

These limits have led certain authors to complete the Austinian analysis, either by highlighting the importance of the interlocutors' subjectivity (as Grice and Searle for philosophy and Butler for sociology do) or by noting the need to take social and cultural context into account (Bourdieu). But these critiques simply continued Austin's error by accepting an insurmountable boundary between discourse and that which lies beyond it (either in the form of the psychology of subjects or of society). (ebd., S. 352f., Anm. 6)

Obwohl sich in diesem Zitat einige Missverständnisse und Ungenauigkeiten hinsichtlich der referierten Positionen offenbaren, lässt sich - und darauf kommt es hier an - erkennen, worauf Callon aus ist: Es geht ihm darum, einen anderen Zugang zum Verhältnis von Diskurs und Materialität zu finden, der weder auf die materiellen Wirkungen von Sprache (Butler) noch auf die Bedeutung sozialer Kontexte (Bourdieu) abstellt. Im Sinne der Akteur-Netzwerk-Theorie fordert er eine Neuausrichtung, die zwar von der Verschränkung von Aussage und Kontext ausgeht, dabei aber ein anderes Verständnis des Kontextes zugrunde legt, das auch Materialitäten und Technik mit einbezieht:

To extend Austin, we have to go in another direction and question the actualization of the contexts and subjectivities that are implied by the utterance. In the case of science, this implies the study of the relationships between SES [singular existential statements; L. G.] and sociotechnical arrangements (agencements). The critique of Austin should not exclude the notion of performativity but rather should enrich and complete it, first, by insisting more on the fact that the context of enunciation is included in the enunciation (semiotic turn) and, 
second, by taking into account the materialities composing that context (ANT turn). (ebd., S. 353, Anm. 6)

Diese hier benannte doppelte Wende ist entscheidend. Sie zielt darauf, Sprache und Materialität nicht gegeneinander auszuspielen, sondern ihre gegenseitige Ko-Konstruktion zu verfolgen. Dabei geht es nicht darum, eine Aussage auf ihren sozialen Kontext zurückzuführen und damit die sprachliche Wirkmächtigkeit als soziale Wirkmächtigkeit zu entlarven. Ähnlich wie Barad zielt Callon vielmehr auf ein wechselseitiges, koevolutives Modell, das Technik, Artefakte und Apparaturen explizit miteinbezieht. Die Grundannahme dieses Unterfangens verdeutlicht sich im von Callon aufgegriffenen und auf Gilles Deleuze zurückgehenden Begriff des ,agencements“, bei dem es gleichermaßen um Diskurs, Text, Aussagen, Modelle wie auch um Instrumente, Techniken, Apparate und Infrastrukturen geht. ,The word agencement has the advantage of being close to the notion of agency: an agencement acts, that is, it transforms a situation by producing differences. The modifier, sociotechnical ' underscores the fact that the entities which are included in the agencement and participate in the actions undertaken are both humans and non-humans." (Callon 2008, S. 38; Hervorh. i. Orig.; s. a. 2009, S. 25 f.)

Der Performativitätsbegriff wird dementsprechend nicht auf sprachlich verfasste (und dann in ihrer Wirkung analysierbare) Äußerungen bezogen, sondern auf heterogene Komplexe verschiedener, aber eben auch sprachlicher Elemente. Obwohl diese Ergänzung umständlich erscheinen mag, ergibt sie sich nach Callon zwingend aus der Verbindung zweier für ihn unumgehbarer Einsichten: der von Austin, dass Aussagen nicht länger von ihren Kontexten zu trennen sind; sowie der der ANT, dass diese Kontexte auch aus nichtmenschlichen Entitäten bestehen (Callon und Latour 2006; Latour 2006). Für ihn erweist sich als zentrales Problem der sozial- und sprachwissenschaftlichen Performativitätsdebatte, dass die Betonung der Wirksamkeit von Sprache und Diskursen dazu führt, die Mitwirkung des Nichtsprachlichen an der sprachlichen Äußerung aus dem Blick zu verlieren. Weil ihm zugleich aber auch Bourdieus Ansatz als defizitär erscheint, ${ }^{18}$ betont er stattdessen, ähnlich wie Barad, dass der Performative Turn asymmetrisch bleibt, solange das Nichtsprachliche lediglich als Einschreibefläche sprachlicher Materialisierungen - und damit selbst als formbares, passives Material - begriffen wird.

Obwohl sie sich in einigen Hinsichten unterscheiden, können die Positionen von Barad und Callon demnach als komplementär begriffen werden: Während Barad stärker auf ein neues Verständnis von Materialität hinarbeitet und dementsprechend betont, dass auch das Materielle selbst schon als performativ begriffen werden muss, arbeitet sich Callon stärker am soziologischen Verständnis des Kontextes (der Äußerung) ab und argumentiert dafür, dass das Materielle und Technische hier immer schon am Vollzugsprozess beteiligt ist - der entsprechend symmetrisch analysiert werden muss. Trotz unterschiedlicher Schwerpunkte und Begrifflichkeiten geht es

\footnotetext{
18 Aus Sicht der ANT erweisen sich hier insbesondere das Verständnis des Sozialen sowie das damit verbundene Konzept des Kontextes als problematisch. Kritisiert wird vor allem der Soziozentrismus und der Reduktionismus dieser soziologischen Perspektive (Callon 1998, 2007, S. 326f.; Latour 1988, S. 253, Anm. 15, 2002; Gertenbach 2018, S. 268 ff.).
} 
aber beiden um heterogene Verflechtungen. Ihre zentralen Begriffe - Intraaktivität, „agencement", „entanglements" etc. - machen deutlich, dass die Ausrichtung an Relationen und Verwicklungen vor allem darauf zielt, ein andersartiges Modell an die Stelle der Dualismen von Diskurs und Materialität, Wort und Welt bzw. Zeichen und Ding treten zu lassen. Die hiermit angedeutete paradigmatische Differenz lässt sich nun abschließend genauer in den Blick nehmen.

\section{Performativität zwischen Sprach- und Medienparadigma}

Angesichts der Vielschichtigkeit und der Unterschiede zwischen den sich auf den Performativitätsbegriff stützenden Positionen läge es nahe, sich der Kritik an der Ubiquität und Profillosigkeit der „,catch-all-Phrase“ Performativität anzuschließen. Die Diskussion der verschiedenen Positionen sollte aber nicht nur ein Bild der zahlreichen Verzweigungen der Performativitätsdebatte liefern. Sie sollte auch eine Grundlage bieten, um diese Debatte genauer zu sortieren. Dies soll nun abschlieBend unternommen werden. Als Ausgangspunkt kann dabei die Beobachtung dienen, dass der Begriff sowohl von Positionen herangezogen wird, denen es um die wirklichkeitsgenerierende Kraft der Sprache geht, als auch von solchen, die sich von der Dominanz des Lingustic Turns befreien wollen. Dies ist bemerkenswert, weil der Performative Turn somit zugleich als Vollendung wie auch als Verabschiedung des Linguistic Turns begriffen werden kann. Anstatt diese Ambiguität über begriffliche Eingrenzungen (vermeintlich) aufzulösen, gilt es, die darin enthaltenen konzeptionellen und theorielogischen Differenzen in den Blick zu nehmen. Denn die Heterogenität der Performativitätstheorien lässt sich auf die Präsenz verschiedener Paradigmen in den Sozial- und Kulturwissenschaften beziehen. Eine Möglichkeit zur Sortierung dieser Paradigmen bietet sich im Anschluss an die medientheoretischen Schriften von Sybille Krämer (2001, 2002, 2008). Dort versucht sie nicht nur, Performativität als Medialität neu zu rekonstruieren (Krämer 2002, S. 329 ff.). Sie schlägt in ihrer Medientheorie auch eine Unterscheidung vor, die sich zur Systematisierung der verschiedenen Performativitätstheorien aufgreifen lässt.

Grundlage hierfür ist, dass Krämer die Medientheorie nicht über einen vermeintlich klar benennbaren Gegenstand (,die Medien“) bestimmt. Interessiert an der Differenz zwischen Sprach- und Medientheorie argumentiert sie vielmehr dafür, deren Besonderheit in einem grundlegenden Perspektivenwechsel zu sehen - nämlich von einer semiologischen zu einer mediologischen Perspektive. Beide Perspektiven enthalten jeweils eigene Fragestellungen und theoretische Annahmen (Krämer 2008, S. $33 \mathrm{ff}$.), die sich in die hier relevante Fragestellung wie folgt übersetzen lassen: Die mit den klassischen Varianten der Sprachtheorie verbundene und am Modell der Schrift orientierte semiologische Perspektive lässt sich dadurch bestimmen, dass sie sich am Topos der Repräsentation abarbeitet. Ihre im Hintergrund stehende Grundfrage betrifft dann das zeichentheoretische Problem der Referenz, d. h. die Frage, für was das Zeichen Zeichen ist. Die mit neueren Medientheorien verbundene mediologische Perspektive hingegen kann dadurch charakterisiert werden, dass sie am Topos 
der Vermittlung ausgerichtet ist. An die Stelle der Differenz zwischen Zeichen und Objekt tritt damit die Orientierung am heterogenen Prozess der Mediatisierung. ${ }^{19}$

Mit dieser Unterscheidung können nun die diskutierten Performativitätstheorien in ihrer systematischen Differenz genauer bestimmt werden, weil hiermit eine sprachtheoretische (dem semiologischen Modell folgende) von einer medientheoretischen (dem mediologischen Modell folgenden) Perspektive auf Performativität unterschieden werden kann. Dabei zeigt sich, dass die rekonstruierten Soziologien des Performativen für ein sprachtheoretisches Modell stehen, das in seiner Weiterführung der Sprechakttheorie auch deren Fragestellungen und zentrale Theoriefiguren weiterführt. Dies gilt für Bourdieu, geradezu paradigmatisch aber vor allem für Butler. Denn auch wenn Sprache bei ihr stets mit Körperlichkeit verbunden ist und nicht mehr repräsentationslogisch konzipiert wird, sind die Problemstellungen des sprachtheoretischen Modells von Performativität noch überaus präsent - in der referentiellen Frage nach dem durch Sprache bezeichneten Objekt sowie dem konstitutiv vom Diskurs her gedachten Prozess der Materialisierung. Hinzu kommt bei beiden ein Folgeproblem, das sich gerade durch die praxistheoretische Umschreibung von Sprache ergibt. Sie führt nämlich dazu, dass auch das sprachliche Darstellen nach dem Modell des Erzeugens gedacht wird. Dies ist nicht nur problematisch, weil damit die Differenz zwischen dem technischen Erzeugen und dem symbolischen Darstellen nivelliert wird, sondern auch, weil damit ein fragwürdiger Anthropozentrismus bestärkt wird. Wiederum zeigt sich insbesondere bei Butler, dass der Performative Turn, obwohl er

in vieler Hinsicht anknüpft an die subjektkritischen Bestrebungen im 20. Jahrhundert, gleichwohl an der Idee der Erzeugung, der Konstruktion und Konstitution als fortdauerndem Nukleus menschlicher Kulturleistung [partizipiert]. Indem das Sprechen als ein Handeln, indem symbolische Darstellungen als Herstellungen von Welt begriffen werden, wird das demiurgische Potenzial, das den instrumentellen Praktiken inne wohnt, nun auf die semiotischen Praktiken übertragen: nicht nur technische Handlungen, auch ,Zeichenhandlungen“ werden damit zur Springquelle weltschöpferischen Tuns. Herstellen und Darstellen verschwistern sich. (Krämer 2011, S. 182)

Diese Kritik von Krämer macht deutlich, dass die Differenzierung von Sprachund Medienparadigma dazu beitragen kann, derartige Folgeprobleme genauer in den Blick zu nehmen. An Butler wird so erkennbar, dass sie trotz der offenkundigen Kritik an klassischen Handlungstheorien eine vom sprachtheoretischen Modell übernommene Problemstellung weiterführt.

Im Unterschied zur Sprechakttheorie und zu Bourdieu und Butler lassen sich Barad und Callon hingegen als paradigmatische Vertreter*innen einer medientheo-

\footnotetext{
19 Die vorliegenden Überlegungen schließen an Krämer (2008, S. 33 ff.) an, akzentuieren deren Ausführungen aber ausgehend von den skizzierten Performativitätstheorien anders. In Krämers instruktiver Diskussion der Medientheorie geht es stärker denn an dieser Stelle um die Verkehrung des Verhältnisses von Sichtbarkeit und Unsichtbarkeit sowie um die Differenz zwischen der (semiologischen) Frage nach der Bedeutung des Zeichens und der (mediologischen) Frage nach der Materialität der Medien. Zentral ist aber auch bei ihr die Bestimmung der Medientheorie als Theorie der Vermittlung und Übertragung, insbesondere über die Figur des Mittlers und des Boten.
} 
retischen Perspektive auf Performativität begreifen. Dies zeigt sich daran, dass ihr Ausgangspunkt nicht die Wirkmächtigkeit der Sprache oder die Formbarkeit des Materiellen durch Sprache, sondern die Koexistenz und Verschränkung heterogener Elemente - Diskurs, Materialität, Technik, etc. - ist. Anstatt den Fokus auf die Benennungs- und Klassifikationsmacht des Sprachlichen oder des durch Sprache hindurch wirkenden Sozialen zu richten, setzen sie am Prozess der Mediatisierung, d.h. an vielfältigen Vermischungen und Vermittlungen an. Eine solche genuin mediologische Perspektive ersetzt die Frage danach, für was das Zeichen Zeichen ist, durch die Frage, wie Wörter und Dinge miteinander vermittelt und ineinander verschränkt sind, wie die Begriffe, die Callon und Barad in die Performativitätsdebatte einführen, deutlich machen. Obwohl natürlich auch bei Callon und Barad Diskurse, Sprache und Äußerungen eine Rolle spielen, werden sie aus einer strukturell anderen Perspektive als etwa bei Bourdieu und Butler untersucht (vgl. Callon 2009, S. 18).

Weil sich hierin eine wesentliche Differenz zu den sprachtheoretisch argumentierenden Performativitätsansätzen zeigt, möchte ich vorschlagen, zwischen einem semiologischen und einem mediologischen Performativitätskonzept zu unterscheiden. Dies ermöglicht nicht nur eine systematischere Diskussion der unter dem Label des Performative Turns subsumierten Positionen. Es weist auch einen Weg, den hier beispielhaft an Barad und Callon diskutierten Positionen besser gerecht werden zu können als dies bisher der Fall war. Denn es eröffnet einen zweiten Blick auf einige bis heute virulente Missverständnisse gegenüber deren Schriften. Gerade an Callon, der ja immerhin als Begründer der Performativitätsforschung in der neueren Wirtschaftssoziologie gilt, lässt sich dies abschließend kurz umreißen.

Ein wesentlicher Effekt der Unterscheidung zweier Paradigmen der Performativitätsforschung ist es, die nun als medientheoretisch gefassten Positionen als differenten und demnach eigenständigen Ansatz ernstnehmen zu können. Dementsprechend ist $\mathrm{zu}$ vermuten, dass deren Besonderheit und damit auch deren Gehalt solange unberücksichtigt oder missverstanden bleiben, wie sie auf der Basis der Frage- und Problemstellungen der sprachtheoretischen Perspektive diskutiert werden. Gerade die Diskussionen um Callon zeigen dies sehr anschaulich, weil genau diese andersartige Grundlegung des Konzepts der Performativität immer wieder übersehen wurde. Obwohl er - als Mitbegründer der ANT wenig überraschend - in seinen Texten betont, dass Sprache nicht von ihrem Kontext zu trennen ist und Aussagen nur als Teil von soziotechnischen Arrangements zu untersuchen sind, wurde er vor allem in der wirtschaftssoziologischen Performativitätsdebatte häufig dafür kritisiert, dass er die Wirklichkeit als unmittelbaren Effekt sprachlicher Aussagen begreife und wirtschaftliches Handeln oder gar die Ökonomie als solche aus der Ökonomik, d. h. der performativen Kraft der wirtschaftswissenschaftlichen Theorien herleite (MacKenzie und Millo 2003; Santos und Rodrigues 2009; Sparsam 2018; Vosselman 2014). Die Kritik unterstellt also eine Hypostasierung von Sprache und Theorien, die Callon mit der Weiterentwicklung des Performativitätskonzepts eigentlich überwinden wollte. ${ }^{20}$ Diese Diskrepanz ist nicht allein ungenauen Formulierungen oder

20 Dies gilt auch für den einflussreichen Beitrag von MacKenzie (2004), der Callons weitreichende Performativitätsthese als „Austinian performativity“ bezeichnet und damit - den wesentlichen Punkt verfehlend zu einer Art Neuauflage der klassischen Sprechakttheorie macht. 
oberflächlichen Lektüren zuzuschreiben, sie gründet in der problematischen Annahme, dass Performativitätstheorien notwendigerweise auch Sprachtheorien sein müssen. Um diesen Missverständnissen zu entgehen, hat Callon schließlich vorgeschlagen, den Begriff der Performativität durch den der Performation bzw. der KoPerformation zu ersetzen:

I thought that to describe this approach, the notion of performativity could be useful. To avoid any misinterpretation, a mistake so easy to make (some people have thought that I was saying that the economy was created entirely by economics!), I finally opted for the notion of performation. [...] I moreover recently added that this activity of performation is always caught up in collective activities which don't mobilize only professional economists. That's why I spoke of co-performation. (Callon 2009, S. 19)

Die Unterscheidung zwischen sprachtheoretischen und medientheoretischen Performativitätskonzepten wirft aber nicht nur ein anderes Licht auf die Rezeption der hier als medientheoretisch vorgestellten Positionen. Sie bietet auch eine Möglichkeit, den Performative Turn noch einmal auf neue Weise in den Blick zu nehmen. Dies betrifft vor allem seine konzeptionelle Tragweite und die Behauptung, dass hierin eine zentrale Wende der zeitgenössischen Theorie- und Forschungsentwicklung enthalten ist. Dabei zeigt sich zunächst, dass er trotz der Herkunft aus der Sprechakttheorie mitnichten als Fortsetzung oder auch als Unterform des Linguistic Turns begriffen werden kann. Denn gerade die medientheoretischen Positionen machen ja deutlich, dass der Performativitätsbegriff auch mit der Idee einer umfassenden Neuausrichtung der Sozial- und Kulturtheorien verbunden ist. Weil es nicht nur darum geht, die Forschung lediglich auf Phänomene aufmerksam zu machen, die lange Zeit ausgeblendet oder in den Hintergrund gedrängt wurden, ist die Behauptung eines „Turns“ in programmatischer Hinsicht durchaus angemessen.

Die Frage, die sich dann aber mit Blick auf die medientheoretische Perspektive stellt, ist, wie sich der Performative Turn zu den zahlreichen anderen mittlerweile ausgerufenen Turns - „material“, ,iconic“, ,,sensory“, ,emotional“, „,ontological“, „spatial“, „vital“, „visual“, „,body“ etc. - der Sozial- und Kulturtheorie verhält. Dem ersten Anschein nach handelt es sich hierbei um heterogene und miteinander konkurrierende Theorie- und Forschungsprogramme. Aus der Diskussion des Performative Turns lässt sich jedoch ein anderes Argument entwickeln: Denn letztlich zeigen gerade die hier als medientheoretisch bezeichneten Theorieansätze, dass der Performativitätsbegriff nicht im Widerspruch zu diesen anderen Turns steht, sondern sich mit einigen durchaus produktiv verbinden lässt - etwa über die Betonung von Materialität oder die Kritik an der Priorisierung von Sprache. Ohne das gegenwärtige Theoriefeld der Sozial- und Kulturwissenschaften dadurch mit einem neuen Masternarrativ zu überziehen oder sich auf die Suche nach dem „Turn der Turns“ (Fischer 2015; Bachmann-Medick und Buden 2008, S. 35) zu begeben, wäre also danach zu fragen, ob hierin nicht eine umfassendere Theoriedynamik zum Ausdruck kommt und die unterschiedlichen Forschungsprogramme und Turns nicht zumindest 
über gemeinsame Fluchtlinien verfügen. ${ }^{21}$ In diesem Sinne wäre zu hoffen, dass die oben erfolgte Diskussion des Performative Turns nicht nur zu einer präziseren Auseinandersetzung mit den verschiedenen Performativitätstheorien beitragen kann, sondern auch dabei behilflich ist, derartige Dynamiken der auf den ersten Blick doch recht heterogenen Forschungs- und Theorielandschaft der Soziologie genauer $\mathrm{zu}$ identifizieren.

Funding Open Access funding enabled and organized by Projekt DEAL.

Open Access Dieser Artikel wird unter der Creative Commons Namensnennung 4.0 International Lizenz veröffentlicht, welche die Nutzung, Vervielfältigung, Bearbeitung, Verbreitung und Wiedergabe in jeglichem Medium und Format erlaubt, sofern Sie den/die ursprünglichen Autor(en) und die Quelle ordnungsgemäß nennen, einen Link zur Creative Commons Lizenz beifügen und angeben, ob Änderungen vorgenommen wurden.

Die in diesem Artikel enthaltenen Bilder und sonstiges Drittmaterial unterliegen ebenfalls der genannten Creative Commons Lizenz, sofern sich aus der Abbildungslegende nichts anderes ergibt. Sofern das betreffende Material nicht unter der genannten Creative Commons Lizenz steht und die betreffende Handlung nicht nach gesetzlichen Vorschriften erlaubt ist, ist für die oben aufgeführten Weiterverwendungen des Materials die Einwilligung des jeweiligen Rechteinhabers einzuholen.

Weitere Details zur Lizenz entnehmen Sie bitte der Lizenzinformation auf http://creativecommons.org/ licenses/by/4.0/deed.de.

\section{Literatur}

Ahmed, S. (2008). Imaginary prohibitions: Some preliminary remarks on the founding gestures of the „New Materialism“. European Journal of Women's Studies, 15(1), 23-39.

Austin, J. L. (1946). Other Minds. Proceedings of the Aristotelian Society. Supplementary volumes, logic and reality, 20, 148-187.

Austin, J. L. (1962). How to do things with words. Oxford: Oxford University Press.

Austin, J. L. (1968). Performative und konstatierende Äußerung. In R. Bubner (Hrsg.), Sprache und Analysis. Texte zur englischen Philosophie der Gegenwart (S. 140-153). Göttingen: Vandenhoeck \& Ruprecht.

Austin, J. L. (1986). Performative Äußerungen. In J. L. Austin, Gesammelte philosophische Aufsätze (S. 305-327). Stuttgart: Reclam.

Austin, J. L. (2002). Zur Theorie der Sprechakte. Stuttgart: Reclam.

Bachmann-Medick, D., \& Buden, B. (2008). Kulturwissenschaften - Eine Übersetzungsperspektive. Doris Bachmann-Medick im Gespräch mit Boris Buden. In B. Buden, S. Nowotny (Hrsg.), Übersetzung: Das Versprechen eines Begriffs (S. 29-42). Wien: Turia + Kant.

Bal, M. (2001). Performanz und Performativität. In J. Huber (Hrsg.), Interventionen 10: Kultur - Analysen (S. 197-241). Wien: Springer/Edition Voldemeer.

Barad, K. (2003). Posthumanist performativity. Toward an understanding of how matter comes to matter. Journal of Women in Culture and Society, 28(3), 801-831.

Barad, K. (2007). Meeting the universe halfway. Quantum physics and the entanglement of matter and meaning. Durham \& London: Duke University Press.

Barad, K. (2012a). Agentieller Realismus. Über die Bedeutung materiell-diskursiver Praktiken. Berlin: Suhrkamp.

Barad, K. (2012b). Nature's queer performativity. Kvinder, Køn og forskning/Women, Gender and Research, 2(1-2), 25-53.

Bauman, Z. (2009). Gemeinschaften. Auf der Suche nach Sicherheit in einer bedrohlichen Welt. Frankfurt a. M.: Suhrkamp.

${ }^{21}$ Mit einer anderen Schwerpunktsetzung kann hier auch von einer postkonstruktivistischen Tendenz der Sozial- und Kulturtheorie gesprochen werden (vgl. Gertenbach 2017). 
Boldyrev, I., \& Svetlova, E. (2016). After the turn: How the performativity of economics matters. In I. Boldyrev \& E. Svetlova (Hrsg.), Enacting dismal science. New perspectives on the performativity of economics (S. 1-27). New York: Palgrave Macmillan.

Bourdieu, P. (1991). Language and symbolic power. Cambridge: Polity Press.

Bourdieu, P. (1992). Die verborgenen Mechanismen der Macht. Hamburg: VSA.

Bourdieu, P. (2015). Was heißt sprechen? Zur Ökonomie des sprachlichen Tausches. Wien: New Academic Press.

Bourdieu, P., \& Wacquant, L. (1996). Reflexive Anthropologie. Frankfurt a. M.: Suhrkamp.

Butler, J. (1995). Für ein sorgfältiges Lesen. In S. Benhabib, J. Butler, D. Cornell \& N. Fraser (Hrsg.), Der Streit um Differenz. Feminismus und Postmoderne in der Gegenwart (S. 122-132). Frankfurt a. M.: Fischer.

Butler, J. (1997). Körper von Gewicht. Die diskursiven Grenzen des Geschlechts. Frankfurt a. M.: Suhrkamp.

Butler, J. (1999). Performativity's social magic. In R. Shusterman (Hrsg.), Bourdieu: A critical reader (S. 113-128). Malden/Oxford: Blackwell.

Butler, J. (2002). Psyche der Macht. Das Subjekt der Unterwerfung. Frankfurt a. M.: Suhrkamp.

Butler, J. (2006). Haß spricht. Zur Politik des Performativen. Frankfurt a. M.: Suhrkamp.

Butler, J. (2015). Notes toward a performative theory of assembly. Cambridge, Mass./London: Harvard University Press.

Callon, M. (1998). Introduction: The embeddedness of economic markets in economics. In M. Callon (Hrsg.), The laws of the markets (S. 1-57). Oxford: Blackwell.

Callon, M. (2004). Actor-network theory - the market test. In J. Law \& J. Hassard (Hrsg.), Actor network theory and after (S. 181-195). Oxford: Blackwell.

Callon, M. (2007). What does it mean to say that economics is performative? In D. MacKenzie, F. Muniesa \& L. Siu (Hrsg.), Do economists make markets? On the performativity of economics (S. 311-357). Princeton: Princeton University Press.

Callon, M. (2008). Economic markets and the rise of interactive agencements: From prosthetic agencies to habilitated agencies. In T. J. Pinch \& R. Swedberg (Hrsg.), Living in a material world. Economic sociology meets science and technology studies (S. 29-56). Cambridge: MIT Press.

Callon, M. (2009). Elaborating the notion of performativity. Le Libellio d'Aegis, 5(1), 18-29.

Callon, M., \& Latour, B. (2006). Die Demontage des großen Leviathans. Wie Akteure die Makrostruktur ihrer Realität bestimmen und Soziologen ihnen dabei helfen. In A. Belliger \& D. J. Krieger (Hrsg.), ANThology. Ein einführendes Handbuch zur Akteur-Netzwerk-Theorie (S. 75-101). Bielefeld: transcript.

Callon, M., Barry, A., \& Slater, D. (2002). Technology, politics and the market: An interview with Michel Callon. Economy and Society, 31(2), 285-306.

Carlson, M. (2004). Performance. A critical introduction. New York: Routledge.

Derrida, J. (1999). Signatur - Ereignis - Kontext. In J. Derrida, Randgänge der Philosophie (S. 325-351). Wien: Passagen.

Derrida, J. (2002). Unabhängigkeitserklärungen. In U. Wirth (Hrsg.), Performanz. Zwischen Sprachphilosophie und Kulturwissenschaften (S. 121-128). Frankfurt a. M.: Suhrkamp.

Eickelmann, J. (2017). „Hate Speech“ und Verletzbarkeit im digitalen Zeitalter. Phänomene mediatisierter Missachtung aus Perspektive der Gender Media Studies. Bielefeld: transcript.

Felman, S. (2003). The scandal of the speaking body. Don Juan with J. L. Austin, or seduction in two languages. Stanford: Stanford University Press.

Fischer, J. (2015). Simmels Sinn der Sinne. Zum vital turn der Soziologie. In H. Göbel, S. Prinz (Hrsg.), Die Sinnlichkeit des Sozialen. Wahrnehmung und materielle Kultur (S. 423-440). Bielefeld: transcript.

Fischer-Lichte, E. (1998). Auf dem Wege zu einer performativen Kultur. In Paragrana. Internationale Zeitschrift für Historische Anthropologie. Band 7: Kulturen des Performativen. (S. 13-29). Berlin: Akademie Verlag.

Franck, G. (1998). Ökonomie der Aufmerksamkeit. Ein Entwurf. Wien: Hanser.

Gertenbach, L. (2017). Postkonstruktivismus in der Kultursoziologie. In S. Moebius, F. Nungesser \& K. Scherke (Hrsg.), Handbuch Kultursoziologie (S. 1-24). Wiesbaden: Springer VS.

Gertenbach, L. (2018). Die Droge als Aktant: Akteur-Netzwerk-Theorie. In R. Feustel, H. Schmidt-Semisch \& U. Bröckling (Hrsg.), Handbuch Drogen in sozial- und kulturwissenschaftlicher Perspektive (S. 263-277). Wiesbaden: Springer VS.

Gertenbach, L., \& Laux, H. (2018). Zur Aktualität von Bruno Latour. Einleitung in sein Werk. Wiesbaden: Springer VS. 
Gond, J.-P., Cabantous, L., Harding, N., \& Learmonth, M. (2016). What do we mean by performativity in organizational and management theory? The uses and abuses of performativity. International Journal of Management Reviews, 18(4), 440-463.

Gonquergood, D. (1989). Poetics, play, process, and power: The performative turn in anthropology. Text and Performance Quarterly, 9(1), 82-88.

Hempfer, K. W. (2011). Performance, Performanz, Performativität. Einige Unterscheidungen zur Ausdifferenzierung eines Theoriefeldes. In K. W. Hempfer \& J. Volbers (Hrsg.), Theorien des Performativen. Sprache, Wissen, Praxis. Eine kritische Bestandsaufnahme (S. 13-41). Bielefeld: transcript.

Hempfer, K. W., \& Volbers, J. (Hrsg.). (2011). Theorien des Performativen. Sprache, Wissen, Praxis. Eine kritische Bestandsaufnahme. Bielefeld: transcript.

Hoppe, K., \& Lemke, T. (2015). Die Macht der Materie. Grundlagen und Grenzen des agentiellen Realismus von Karen Barad. Soziale Welt. Zeitschrift für sozialwissenschaftliche Forschung und Praxis, 3(66), 261-280.

Kertscher, J., \& Mersch, D. (Hrsg.). (2003). Performativität und Praxis. München: Fink.

Krämer, S. (2001). Sprache, Sprechakt, Kommunikation. Sprachtheoretische Positionen des 20. Jahrhunderts. Frankfurt a. M.: Suhrkamp.

Krämer, S. (2002). Sprache - Stimme - Schrift: Sieben Gedanken über Performativität als Medialität. In U. Wirth (Hrsg.), Performanz. Zwischen Sprachphilosophie und Kulturwissenschaften (S. 323-346). Frankfurt a. M.: Suhrkamp.

Krämer, S. (2003). Was tut Austin, indem er über das Performative spricht? Ein anderer Blick auf die Anfänge der Sprechakttheorie. In J. Kertscher \& D. Mersch (Hrsg.), Performativität und Praxis (S. 19-33). München: Fink.

Krämer, S. (2008). Medium, Bote, Übertragung. Kleine Metaphysik der Medialität. Frankfurt a. M.: Suhrkamp.

Krämer, S. (2011). Windungen und Wendungen geisteswissenschaftlicher Debatten: Ein Kommentar zu den Grenzen des ,,performative turn“, „,media turn“ und ,iconic turn“. In M. Grizelj \& O. Jahraus (Hrsg.), Theorietheorie. Wider die Theoriemüdigkeit in den Geisteswissenschaften (S. 181-195). München: Fink.

Latour, B. (1988). The pasteurization of France. Cambridge, Mass.: Harvard University Press.

Latour, B. (2002). Der Blutkreislauf der Wissenschaft. Joliots wissenschaftliche Intelligenz als Beispiel. In B. Latour, Die Hoffnung der Pandora. Untersuchungen zur Wirklichkeit der Wissenschaft (S. 96-136). Frankfurt a. M.: Suhrkamp.

Latour, B. (2006). Über technische Vermittlung. Philosophie, Soziologie und Genealogie. In A. Belliger \& D. J. Krieger (Hrsg.), ANThology. Ein einführendes Handbuch zur Akteur-Netzwerk-Theorie (S. 483-528). Bielefeld: transcript.

Lovell, T. (2000). Thinking feminism with and against Bourdieu. Feminist Theory, 1(1), 11-32.

Loxley, J. (2007). Performativity. London: Routledge.

Lyotard, J.-F. (1979). La condition postmoderne. Rapport sur le savoir. Paris: Les Editions de Minuit.

Lyotard, J.-F. (1986). Das postmoderne Wissen. Ein Bericht (vollst. überarb. Fassung). Graz/Wien: Böhlau.

MacKenzie, D. (2004). The big, bad wolf and the rational market: Portfolio insurance, the $1987 \mathrm{crash}$ and the performativity of economics. Economy and Society, 33(3), 303-334.

MacKenzie, D. (2006). Is economics performative? Option theory and the construction of derivatives markets. Journal of the History of Economic Thought, 28(1), 29-55.

MacKenzie, D., \& Millo, Y. (2003). Constructing a market, performing theory: The historical sociology of a financial derivatives exchange. American Journal of Sociology, 109(1), 107-145.

Martschukat, J., \& Patzold, S. (2003). Geschichtswissenschaft und ,„performative turn“. Eine Einführung in Fragestellungen, Konzepte und Literatur. In J. Martschukat \& S. Patzold (Hrsg.), Geschichtswissenschaft und „performative turn“. Ritual, Inszenierung und Performanz vom Mittelalter bis zur Neuzeit (S. 1-31). Köln/Weimar/Wien: Böhlau.

Medina, J. (2010). The performative turn and the emergence of post-analytic philosophy. In R. Braidotti (Hrsg.), After poststructuralism: Transitions and transformations (S. 275-305). Durham: Acumen.

Müller, J. (2015). Bestimmbare Unbestimmtheiten. Skizze einer indeterministischen Soziologie. Paderborn: Fink.

Muniesa, F. (2014). The provoked economy. Economic reality and the performative turn. London: Routledge.

Pefanis, G. P. (2017). Performative and moral issues in the theatrical metaphor. Itinera, 13, 93-108.

Pickering, A. (1995). The mangle of practice. Time, agency, and science. Chicago: University of Chicago Press. 
Reckwitz, A. (2017). Die Gesellschaft der Singularitäten. Zum Strukturwandel der Moderne. Berlin: Suhrkamp.

Santos, A. C., \& Rodrigues, J. (2009). Economics as social engineering? Questioning the performativity thesis. Cambridge Journal of Economics, 33(5), 985-1000.

Schäfer, H. (Hrsg.). (2016). Praxistheorie. Ein soziologisches Forschungsprogramm. Bielefeld: transcript.

Searle, J. R. (1969). Speech acts. An essay in the philosophy of language. New York: Cambridge University Press.

Searle, J. R. (1989). How performatives work. Linguistics and Philosophy, 12, 535-558.

Sparsam, J. (2018). Wie ökonomisches Wissen wirksam wird. Von der Performativitäts- zur Verwendungsforschung. Wiesbaden: Springer VS.

Volbers, J. (2014). Performative Kultur. Eine Einführung. Wiesbaden: Springer VS.

Vosselman, E. (2014). The ,performativity thesis“ and its critics: Towards a relational ontology of management accounting. Accounting and Business Research, 44(2), 181-203.

Wirth, U. (2009). Der Performanzbegriff im Spannungsfeld von Illokution, Iteration und Indexikalität. In U. Wirth (Hrsg.), Performanz. Zwischen Sprachwissenschaft und Kulturwissenschaften (S. 9-60). Frankfurt a. M.: Suhrkamp.

Lars Gertenbach geb. 1979. Dr. phil., wissenschaftlicher Mitarbeiter an der Universität Kassel. Forschungsschwerpunkte: Soziologische Theorie, Kultursoziologie, Science \& Technology Studies, Diskursforschung, Poststrukturalistische Soziologien, Studies of Governmentality, Akteur-Netzwerk-Theorie, Theorien der Gemeinschaft. Ausgewählte Publikationen: Entgrenzungen der Soziologie. Bruno Latour und der Konstruktivismus, 2015; Konnektivität und Zusammenhalt. Von den zwei Soziologien des sozialen Bandes, in: T. Bedorf \& S. Herrmann (Hrsg.), Das soziale Band. Geschichte und Gegenwart eines sozialtheoretischen Grundbegriffs, 2016; Postkonstruktivismus in der Kultursoziologie, in: S. Moebius, F. Nungesser \& K. Scherke (Hrsg.), Handbuch Kultursoziologie. Band 2: Theorien - Methoden - Felder, 2019. 